\title{
$B=5$ Skyrmion as a two-cluster system
}

\author{
Sven Bjarke Gudnason ${ }^{*}$ and Chris Halcrow ${ }^{\dagger}$ \\ Institute of Modern Physics, Chinese Academy of Sciences, Lanzhou 730000, China
}

(Received 26 February 2018; published 7 June 2018)

\begin{abstract}
The classical $B=5$ Skyrmion can be approximated by a two-cluster system in which a $B=1$ Skyrmion is attached to a core $B=4$ Skyrmion. We quantize this system, allowing the $B=1$ to freely orbit the core. The configuration space is 11 dimensional but simplifies significantly after factoring out the overall spin and isospin degrees of freedom. We exactly solve the free quantum problem and then include an interaction potential between the Skyrmions numerically. The resulting energy spectrum is compared to the corresponding nuclei-the helium-5/lithium-5 isodoublet. We find approximate parity doubling not seen in the experimental data. In addition, we fail to obtain the correct ground-state spin. The framework laid out for this two-cluster system can readily be modified for other clusters and in particular for other $B=4 n+1$ nuclei, of which $B=5$ is the simplest example.
\end{abstract}

DOI: 10.1103/PhysRevD.97.125004

\section{INTRODUCTION}

The Skyrme model is a nonlinear theory of pions that admits topologically nontrivial configurations called Skyrmions, labeled by a topological charge $B$. Skyrme's pioneering idea [1] was to identify these configurations with nuclei and the topological charge with baryon number.

The theory captures many phenomenological features of nuclei such as isospin symmetry, $\alpha$ clustering [2], and rotational bands [3] and even contains a version of the liquid drop model [4]. Recently, the model has successfully explained the energy spectrum of carbon-12 and the large root-mean-square charge radius of the Hoyle state [5] and has been able to describe the rich energy spectrum of oxygen-16 [6]. The Skyrme model is attractive theoretically as there are few adjustable parameters in the Lagrangian. Further, all nucleon interactions and dynamics are determined by this initial Lagrangian.

The model can approximately reproduce the low-energy spectrum of all light $(B=1-8)$ nuclei except the ${ }^{5} \mathrm{Li} /{ }^{5} \mathrm{Be}$ isodoublet $[3,7]$. These two nuclei are usually described in the shell model as an inert core (the $\alpha$ particle) plus one orbiting nucleon. In the most basic shell model, the additional nucleon has either spin $1 / 2$ or $3 / 2$. The spinorbit interaction breaks this degeneracy, making the spin$3 / 2$ state energetically favored. Hence, the ground states of

\footnotetext{
*bjarke@impcas.ac.cn

christ@impcas.ac.cn
}

Published by the American Physical Society under the terms of the Creative Commons Attribution 4.0 International license. Further distribution of this work must maintain attribution to the author(s) and the published article's title, journal citation, and DOI. Funded by SCOAP ${ }^{3}$. helium-5 and lithium-5 have spin $3 / 2$. This story is partially mirrored in the Skyrme model. Here, the $B=5$ Skyrmion is approximately described by a $B=4$ Skyrmion plus an additional $B=1$ Skyrmion. In the standard Skyrme model, the clusters merge into a $D_{2}$-symmetric Skyrmion, although it takes little energy to separate them. In modified Skyrme models such as the loosely bound Skyrme model [8-10] and lightly bound Skyrme model [11,12], the $B=5$ Skyrmion is very well approximated by the two-cluster system. There should then be a low-energy manifold of configurations: those in which the $B=1$ orbits the $B=4$ core. Taking account of these degrees of freedom (d.o.f.) allows us to describe the $B=5$ as a $4+1$-cluster system, just like the shell model. The shell model notion of the spinorbit force is not present in the Skyrme model; instead, there is an interaction potential that depends on the internal orientations of the Skyrmion clusters. The link between these ideas was explored in a two-dimensional toy model in Ref. [13].

In this paper, we consider the quantization of the $B=5$ Skyrmion as a two-cluster system. Each cluster is individually allowed to rotate and isorotate. This is the first time such a system has been quantized within the Skyrme model. The resultant spectrum contains the low-energy spin-3/2- and $-1 / 2^{-}$states, though in the wrong order. We also find parity doubling, not seen in experimental data. The unwanted $3 / 2^{+}$and $1 / 2^{+}$states are not allowed by the $D_{2}$-symmetric Skyrmion, which is not included in our configuration space. Its existence should still affect the energy of the states, though the size of this impact is difficult to measure.

Many nuclei can be described as a core + particle system, such as those close to magic nuclei. The $B=5$ is the simplest of these systems. This motivates us to study the system 
carefully, and the framework we develop should apply more broadly, with a few simple modifications. In fact, much of our formalism applies to any two-cluster system. These are used frequently in nuclear physics, from modeling bound nuclei such as lithium-7 [14] to describing scattering between $\alpha$ particles [15] or carbon-12 nuclei [16]. Hence, we believe our work provides an important step toward understanding this wide range of problems within the Skyrme model.

In the next section, we carefully set up our model of the ${ }^{5} \mathrm{He} /{ }^{5} \mathrm{Li}$ isodoublet as a $4+1$ two-cluster system within the Skyrme model. We then quantize the system in Sec. III before dealing with the cubic symmetry of the core in Sec. IV. The methods described in this section are very general and can be applied to any vibrational quantization in the Skyrme model. We then find states with definite parity and discuss some additional symmetries of the configuration space in Sec. V. The energies of the derived wave functions are calculated and compared to experimental data in Sec. VI. The framework we develop in this paper may be applied to a wide range of systems; we suggest several avenues for further work in Sec. VII. Concluding remarks can be found in Sec. VIII.

\section{SKYRME MODEL, OUR CONFIGURATION SPACE, AND RELATIVE COORDINATES}

The variant of the Skyrme model we consider in this paper is the most commonly used version, albeit with a pion mass and the possibility of a loosely bound potential [8]. The Lagrangian density is

$$
\begin{aligned}
\mathcal{L}= & \operatorname{Tr}\left[\frac{1}{2} L_{\mu} L^{\mu}+\frac{1}{16}\left[L_{\mu}, L_{\nu}\right]\left[L^{\mu}, L^{\nu}\right]-m_{1}^{2}\left(\mathbf{1}_{2}-U\right)\right] \\
& -\frac{1}{4} m_{2}^{2}\left(\operatorname{Tr}\left[\mathbf{1}_{2}-U\right]\right)^{2},
\end{aligned}
$$

where $L_{\mu} \equiv U^{\dagger} \partial_{\mu} U$ is the left-invariant $\mathfrak{g} \mathfrak{t}(2)$-valued current, $U$ is the Skyrme field, $m_{1}$ is the pion mass, and $m_{2}$ is a parameter of the loosely bound potential. The Skyrme field is $S U(2)$ valued and can be written in terms of the pion field $\boldsymbol{\pi}(\boldsymbol{x})$ as

$$
U=\mathbf{1}_{2} \sigma+i \boldsymbol{\tau} \cdot \boldsymbol{\pi},
$$

where $\tau$ is the triplet of Pauli matrices and $\sigma$ is an auxiliary field that ensures that $U$ takes values in $S U(2)$. To visualize a Skyrme configuration, we plot a contour of constant energy density. This is then colored to represent the direction of the pion field at that point on the energy contour. The Skyrme field is colored white/black when $\hat{\pi}_{3}= \pm 1$ and red, green, and blue when $\hat{\pi}_{1}+i \hat{\pi}_{2}=\exp (0)$, $\exp (2 i \pi / 3)$, and $\exp (4 i \pi / 3)$, respectively, where $\hat{\boldsymbol{\pi}} \equiv$ $\boldsymbol{\pi} /|\boldsymbol{\pi}|$ is the normalized pion field.

A Skyrmion is a static energy minimizer of (2.1), for a given topological charge $B$. When the loosely bound potential $[8,9]$ is turned off, $m_{2}=0$, the $B=5$ Skyrmion has $D_{2}$ symmetry. As $m_{2}$ is increased, the Skyrmion separates into two clusters. A $B=1$ Skyrmion is gradually detached from a $B=4$ core that acquires approximate cubic symmetry. This process is shown in Fig. 1.

The initial setup we study in this paper is based on the two-cluster system displayed in Fig. 2. Here, a $B=1$ Skyrmion orbits a cubic $B=4$ Skyrmion at a fixed radius. Both clusters are free to rotate, isorotate, and move around one another. We take the $B=4$ to be fixed at the origin. Although we are not in the center-of-mass frame, several other aspects of the problem simplify using this approximation. There are different ways to interpret the model. One is that the configurations, such as the one in Fig. 2, are good approximations to the classical low-energy configurations of the model. This is true for the loosely bound model, as we have seen in Fig. 1. Another way to interpret the model is that we are simply using convenient coordinates to describe a submanifold of the $B=5$ vibrational manifold. In this case, the image in Fig. 2 does not accurately describe the configuration represented by this point in configuration space. Instead, the point corresponds to some deformed version of the configuration, in which the clusters have merged and distorted. Regardless of interpretation, our analysis of the symmetries and quantization of the system will apply. However, the details of the specific moments of inertia and energies will change.

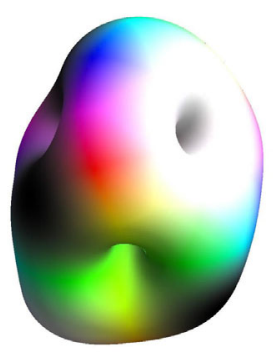

$m_{2}=0$

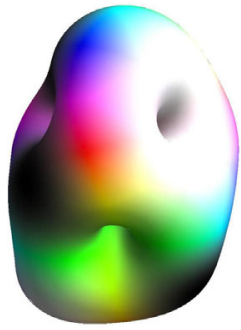

$m_{2}=0.8$

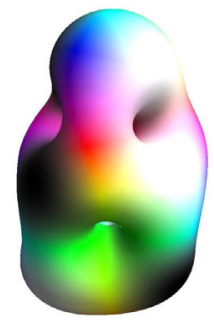

$m_{2}=1.6$

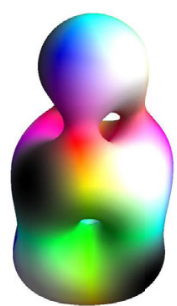

$m_{2}=2.4$

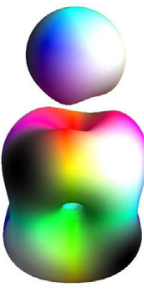

$m_{2}=3.2$

FIG. 1. The $B=5$ Skyrmion with an increasing value of the loosely bound potential parameter $m_{2}$. The leftmost figure corresponds to the standard Skyrme model, while the rightmost figure is the loosely bound Skyrme model with the $B=1$ Skyrmion detached from the cube. The pion mass is taken to be $m_{1}=1$. 


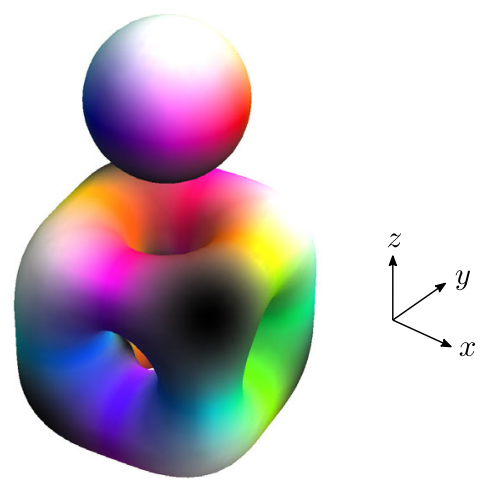

FIG. 2. The basic set up for our model. A $B=1$ Skyrmion orbits a $B=4$ Skyrmion.

Our plan is to solve the free system in which the two clusters do not interact (though are bound together since we have fixed their separation). We will then include the interaction energy by diagonalizing a truncated set of free states with respect to the Hamiltonian, including a numerically generated potential term. This method breaks down if the potential is strong, and hence we are solving the problem in the weak-coupling limit. We will suggest how one may study the strong-coupling limit in Sec. VII. The system is described by 11 coordinates: there are six that specify the orientation of the $B=4$ cluster in space and isospace, three that describe the $B=1$ 's orientation, and the remaining two that specify the orbital position of the $B=1$. This is a formidable number of d.o.f. However, the system transforms simply under overall rotations and isorotations. In particular, the interaction potential is invariant under such transformations. If we can "factor out" these symmetries, only five coordinates, describing the relative interaction between clusters, will remain. This type of reduction is common in the study of comets, many of which are described by twocluster systems bound together by the gravitational force. We closely follow the work of Maciejewski [17], who first solved such a problem.

To proceed, we must introduce notation for the individual momenta of the Skyrmions. We define:

(i) $\boldsymbol{J}^{(i)}$ : space-fixed angular momentum of the $B=i$ Skyrmion.

(ii) $\boldsymbol{L}^{(i)}$ : body-fixed angular momentum of the $B=i$ Skyrmion.

(iii) $\boldsymbol{I}^{(i)}$ : space-fixed isospin of the $B=i$ Skyrmion.

(iv) $\boldsymbol{K}^{(i)}$ : body-fixed isospin of the $B=i$ Skyrmion.

(v) $\boldsymbol{R}$ : position of the $B=1$ Skyrmion relative to the $B=4$, in the space-fixed frame.

(vi) $\boldsymbol{P}$ : orbital angular momentum of the $B=1$ Skyrmion relative to the $B=4$ Skyrmion.

The body-fixed and space-fixed angular and isoangular momenta are related by the orthogonal matrices $A^{(i)}$ and $B^{(i)}$, also known as the attitude matrices. Explicitly,

$$
\boldsymbol{J}^{(i)}=A^{(i)} \boldsymbol{L}^{(i)} \quad \text { and } \quad \boldsymbol{I}^{(i)}=B^{(i)} \boldsymbol{K}^{(i)} .
$$

Further, the momenta of the $B=1$ are related. We use the conventions that the body-fixed momenta are related as $\boldsymbol{L}^{(1)}=-\boldsymbol{K}^{(1)}$. This is the convention used in Ref. [3] but is opposite to that used in Ref. [18]. It implies that the spacefixed momenta obey

$$
\boldsymbol{J}^{(1)}=-A^{(1)} B^{(1) T} \boldsymbol{I}^{(1)} .
$$

One may combine $A^{(1)} B^{(1) T}$ into a single matrix, reflecting the fact that the $B=1$ 's orientation can be parametrized by only three coordinates. We do not do this, to keep the symmetries of the formalism explicit and to emphasize that this method may be applied to any two-cluster system, not just a core + particle system.

The total (iso)angular momentum is the sum of the individual (iso)momenta and the orbital momentum. A simple way to describe these quantities is to go into the body-fixed frame of the $B=4$ Skyrmion. In this frame, the total angular momentum is

$$
\boldsymbol{J}=\boldsymbol{L}^{(4)}+A^{(4) T} A^{(1)} \boldsymbol{L}^{(1)}+A^{(4) T} \boldsymbol{l},
$$

where $\boldsymbol{l}=\boldsymbol{R} \times \boldsymbol{P}$ is the orbital angular momentum in the space-fixed frame. The total isoangular momentum (isospin) is

$$
\boldsymbol{I}=\boldsymbol{K}^{(4)}+B^{(4) T} B^{(1)} \boldsymbol{K}^{(1)} .
$$

As these are body-fixed momenta, they will satisfy the anomalous commutation relations when quantized. These are

$\left[J_{i}, J_{j}\right]=-i \epsilon_{i j k} J_{k}, \quad\left[I_{i}, I_{j}\right]=-i \epsilon_{i j k} I_{k} \quad$ and $\quad\left[J_{i}, I_{j}\right]=0$.

After quantization, the conserved quantities $J$ and $I$ will correspond to the spin and isospin of the nucleus.

We can now define the relative momenta. One has some choice in how to define these, and ours are chosen for their simplicity in describing the Finkelstein-Rubinstein constraints that we meet in the next section. The relative momenta are

$$
\begin{aligned}
& \boldsymbol{S}=A^{(4) T} A^{(1)} \boldsymbol{L}^{(1)}+A^{(4) T} \boldsymbol{l}, \\
& \boldsymbol{T}=\boldsymbol{B}^{(4) T} \boldsymbol{B}^{(1)} \boldsymbol{K}^{(1)} .
\end{aligned}
$$

These act on the $B=1$ Skyrmion, leaving the cubic core unchanged. The momentum $S$ generates a rolling motion of the $B=1$ around the $B=4$, while $\boldsymbol{T}$ acts only on the orientation of the $B=1$. They satisfy the usual commutation relations

$$
\left[S_{i}, S_{j}\right]=i \epsilon_{i j k} S_{k}, \quad\left[T_{i}, T_{j}\right]=i \epsilon_{i j k} T_{k}
$$


and commute with each other and the overall momenta $\boldsymbol{J}$ and $\boldsymbol{I}$. One could think of the relative coordinates as vibrational d.o.f. of the $B=5$ Skyrmion. Then, in the language of Ref. [6], $\boldsymbol{S}$ and $\boldsymbol{T}$ generate the vibrational manifold, while $\boldsymbol{J}$ and $\boldsymbol{I}$ generate the rotational manifold.

The classical configuration space of the system is naively

$$
\mathcal{M}=S O(3)_{J} \times S O(3)_{I} \times \frac{S O(3)_{S} \times S O(3)_{T}}{U(1)} .
$$

The $U(1)$ factor accounts for the fact that there is a degeneracy in the $S / T$ space. This is clear as we only require 5 d.o.f. to describe the position and orientation of the $B=1$ but $S O(3)_{S} \times S O(3)_{T}$ is six dimensional. Explicitly, a simultaneous rotation about the 3 -axis in the $S$ and $T$ spaces leaves the system invariant. We have also not yet accounted for the discrete symmetries of the system. We consider both the $U(1)$ symmetry and the discrete symmetries carefully in the next section.

\section{QUANTIZATION}

Having described the classical configuration space of our model, we can now quantize the system using a semiclassical quantization. This is done by promoting the coordinates on $\mathcal{M}$ to dynamical d.o.f. Each classical momentum becomes a quantum operator, and the four momenta produce quantized spins, which we denote $J, I, S$, and $T$. A general wave function can be written as

$$
|\Psi\rangle=\sum_{j_{3}, i_{3}, s_{3}, t_{3}, s_{3}^{\prime}, t_{3}^{\prime}} a_{j_{3} i_{3} s_{3} t_{3} s_{3}^{\prime} t_{3}^{\prime}}\left|J j_{3} J\right\rangle\left|I i_{3} I\right\rangle\left|S s_{3} s_{3}^{\prime}\right\rangle\left|T t_{3} t_{3}^{\prime}\right\rangle,
$$

where the three labels of each spin state represent the total spin, the projection onto the body-fixed third axis, and the projection onto the space-fixed third axis. For the overall angular momentum and isospin ( $J$ and $I$ ), the space-fixed projections do not affect the structure of the spin state or its energy. Hence, we may set them to any value, and we choose to fix them equal to their total spins. However, the space-fixed projections for the relative momenta do alter the energies, and so we must allow for linear combinations of these as well as the body-fixed projections.

The $U(1)$ degeneracy in the $S / T$ part of the configuration space $\mathcal{M}$ constrains the wave function (3.1). To see how, we put coordinates on this part of the space. These describe the relative orientation between the $B=1$ and $B=4$ Skyrmions. The rolling motion (generated by $S$ ) may be parametrized using Euler angles $(\phi, \theta, \psi)$. We will use the passive Z-Y-Z conventions (applying $\psi$ first, then $\theta$, and finally $\phi$ ). Using these, when $\psi=0$, the remaining coordinates are the usual spherical polars. The internal motion of the $B=1$ (described by $T$ ) can also be parametrized by Euler angles $\left(\alpha, \beta, \gamma^{\prime}\right)$. The spin states can be written in terms of the Euler angles using Wigner-D functions. The relations are

$$
\left|T t_{3} t_{3}^{\prime}\right\rangle=D_{t_{3}^{\prime} t_{3}}^{T}\left(\alpha, \beta, \gamma^{\prime}\right) \quad \text { and } \quad\left|S s_{3} s_{3}^{\prime}\right\rangle=D_{s_{3}^{\prime} s_{3}}^{S}(\phi, \theta, \psi) \text {, }
$$

where

$$
D_{t_{3}^{\prime} t_{3}}^{T}\left(\alpha, \beta, \gamma^{\prime}\right)=e^{i \alpha t_{3}} D_{t_{3}^{\prime} t_{3}}^{T}(0, \beta, 0) e^{i \gamma^{\prime} t_{3}^{\prime}}
$$

The system is invariant under a simultaneous increase in both $\gamma^{\prime}$ and $\psi$, as these coordinates rotate the $B=1$ Skyrmion around the $z$ axis in opposite directions. This leads to a constraint on the space-fixed projections of $S$ and $T$,

$$
\left(\partial_{\gamma^{\prime}}+\partial_{\psi}\right)|\Psi\rangle=|\Psi\rangle \Rightarrow s_{3}^{\prime}=-t_{3}^{\prime},
$$

and so the overall wave function contains the factor

$$
e^{i S_{3}^{\prime} \psi+i t_{3}^{\prime} \gamma^{\prime}}=e^{i t_{3}^{\prime}\left(-\psi+\gamma^{\prime}\right)} \equiv e^{i t_{3}^{\prime} \gamma},
$$

where we have defined a new coordinate, $\gamma$. Using this coordinate is equivalent to setting $\psi=0$ and $\gamma^{\prime}=\gamma$ in (3.2). Hence, the general wave function is given by

$$
\begin{aligned}
|\Psi\rangle= & \sum_{j_{3}, i_{3}, s_{3}, t_{3}, t_{3}^{\prime}} a_{j_{3} i_{3} s_{3} t_{3} t_{3}^{\prime}}\left|J j_{3} J\right\rangle\left|I i_{3} I\right\rangle D_{-t_{3}^{\prime} s_{3}}^{S}(\phi, \theta, 0) \\
& \times D_{t_{3}^{\prime} t_{3}}^{T}(\alpha, \beta, \gamma) .
\end{aligned}
$$

Note that $\left|t_{3}^{\prime}\right|$ can only take values up to $\min (2 S+1,2 T+1)$.

The Finkelstein-Rubinstein (FR) constraints [19] deal with the fact that the $B=5$ Skyrmion is constructed out of five nucleons, and so the system must obey fermionic statistics. The constraints provide restrictions on the allowed wave functions and determine if $J, I, S$, and $T$ are integers or half-integers. This may be determined by considering the rotations physically. Consider a $2 \pi$ rotation about the 1-axis in the $J$ space, as seen in Fig. 3. This rotates the $B=4$ Skyrmion by $2 \pi$, and the $B=1$ Skyrmion rolls around the cube in a circular orbit. Overall, both Skyrmions rotate by $2 \pi$ on their own 1 -axes. Physically, the $B=1$ represents a single nucleon, a fermion, while the $B=4$ represents four nucleons, a boson. Hence, the wave function should transform by -1 under this rotation. Hence, $J$ must be a half-integer. This was already guaranteed by the fact that $B$ is odd. Similar arguments show that the other conserved spins ( $I, S$, and $T$ ) must each be half-integers. Note that all of the spins are conserved in the free system but after inclusion of the potential only $J$ and $I$ will be. States with different $S$ and $T$ values will mix in the nonfree theory. The classical configuration space $\mathcal{M}$ cannot be used to describe systems with half-integer spins. Finkelstein and Rubinstein showed that we must use the double cover of $\mathcal{M}$ instead, and so the quantum configuration space is 


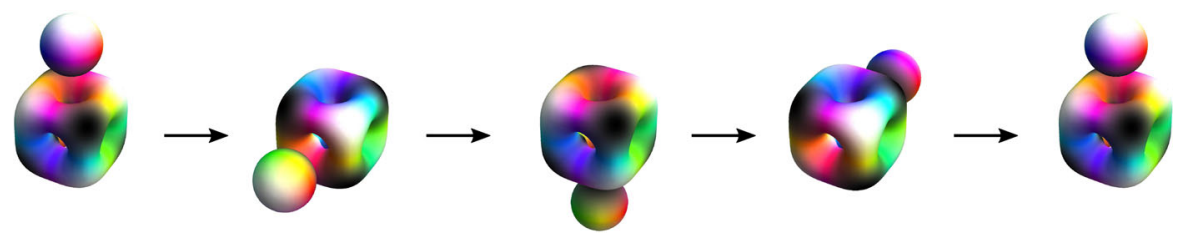

FIG. 3. A $2 \pi$ rotation around the $x$ axis. Note that the $B=1$ Skyrmion does a $2 \pi$ rotation on its own axis and so the wave function should pick up a minus sign under this rotation.

$$
\mathcal{M}_{q}=S U(2)_{J} \times S U(2)_{I} \times \frac{S U(2)_{S} \times S U(2)_{T}}{U(1)}
$$

FR constraints also apply to the discrete symmetries of the configuration space. These are inherited from the discrete symmetries of the clusters. The $B=1$ has no discrete symmetries (and we have already dealt with its continuous symmetry by our parametrization), but the $B=4$ has cubic symmetry. The wave function either transforms trivially or picks up a sign under each element of the symmetry group. To determine which, we may use the algorithm developed in Ref. [18]. The explicit symmetries are most easily expressed in terms of the cube's body-fixed momenta. In these terms, the group is generated by a $C_{4}$ symmetry,

$$
\exp \left(-\frac{\pi i}{2} \hat{L}_{3}^{(4)}-\pi i \hat{K}_{1}^{(4)}\right)|\Psi\rangle=|\Psi\rangle
$$

and a $C_{3}$ symmetry,

$\exp \left(-\frac{2 \pi i}{3 \sqrt{3}}\left(\hat{L}_{1}^{(4)}+\hat{L}_{2}^{(4)}+\hat{L}_{3}^{(4)}\right)-\frac{2 \pi i}{3} \hat{K}_{3}^{(4)}\right)|\Psi\rangle=|\Psi\rangle$.

To find out how these operators act in $\mathcal{M}$, they must be expressed in terms of the new momenta. Our choice of $S$ and $\boldsymbol{T}$ means that the relation is simple. The constraints become

$$
\begin{aligned}
& \exp \left(-\frac{\pi i}{2}\left(\hat{J}_{3}-\hat{S}_{3}\right)-\pi i\left(\hat{I}_{1}-\hat{T}_{1}\right)\right)|\Psi\rangle=|\Psi\rangle \\
& \exp \left(-\frac{2 \pi i}{3 \sqrt{3}}\left(\hat{J}_{1}+\hat{J}_{2}+\hat{J}_{3}-\hat{S}_{1}-\hat{S}_{2}-\hat{S}_{3}\right)-\frac{2 \pi i}{3}\left(\hat{I}_{3}-\hat{T}_{3}\right)\right)|\Psi\rangle \\
& =|\Psi\rangle
\end{aligned}
$$

The form of these operators highlights the fact that applying a rotation to the $B=4$ is equivalent to applying a rotation to the entire two-cluster system, then "undoing" this rotation for the $B=1$. The wave functions of the form (3.6) that satisfy (3.10) and (3.11) are allowed by the symmetries of our system. We call these permissible wave functions. For a fixed $J, I, S$, and $T$, these constraints become a simple linear algebra problem for the coefficients $a$ of (3.6), which one may solve. We will take an alternative approach by using representation theory. This will highlight the similarity between our calculation and other calculations in many subjects such as the Skyrme model [6], molecular vibrations [20], and other nuclear models [21]. Additionally, the method laid out here may be applied to any rotational-vibrational system in the Skyrme model. A reader less interested in our rather technical calculation may wish to skip the next section.

Before proceeding, we comment on the exponentiation of the rotation operators, used in the FR constraints. To evaluate these operators, we must find a matrix representation of the $\mathfrak{s} \mathfrak{u}(2)$ Lie algebras. The $S$ and $T$ operators obey the usual commutation relations (2.10), and so the matrix representation $\boldsymbol{M}$ for the operators $\hat{\boldsymbol{S}}$ and $\hat{\boldsymbol{T}}$ is well known. For example, the spin- $1 / 2$ matrices are simply the Pauli matrices (divided by $1 / 2$ ) and satisfy

$$
\left[M_{i}, M_{j}\right]=i \epsilon_{i j k} M_{k}
$$

However, the $J$ and $I$ operators satisfy the anomalous commutation relations (2.7). Hence, we cannot use the usual $\boldsymbol{M}$. Instead, we use the conjugate representation $\boldsymbol{M}^{*}$, which satisfies

$$
\left[M_{i}^{*}, M_{j}^{*}\right]=-i \epsilon_{i j k} M_{k}^{*}
$$

For example, a $\frac{\pi}{2}$ rotation about the $(0,1,0)$ axis acts on the $S=1 / 2$ wave functions as

$\exp \left(-\frac{\pi i}{2} M_{2}\right)=\exp \left(-\frac{\pi i}{4}\left(\begin{array}{cc}0 & i \\ -i & 0\end{array}\right)\right)=\frac{1}{\sqrt{2}}\left(\begin{array}{cc}1 & 1 \\ -1 & 1\end{array}\right)$,

but the same rotation acts on a $J=1 / 2$ wave function as

$\exp \left(-\frac{\pi i}{2} M_{2}^{*}\right)=\exp \left(-\frac{\pi i}{4}\left(\begin{array}{cc}0 & -i \\ i & 0\end{array}\right)\right)=\frac{1}{\sqrt{2}}\left(\begin{array}{cc}1 & -1 \\ 1 & 1\end{array}\right)$ 


\section{CONSTRUCTING PERMISSIBLE WAVE FUNCTIONS}

In this section, we construct a basis of wave functions that satisfy the constraints (3.10) and (3.11) by splitting the total symmetry group into two parts: one that acts only on the $J / I$ space and the other that acts on the $S / T$ space. Suppose we have a basis of states in the $J / I$ space $\left\{\left|\Psi_{i}\right\rangle\right\}$ and a similar basis in the $S / T$ space $\left\{\Phi_{i}\right\}$. We may act on the $\left|\Psi_{i}\right\rangle$ states using the $J / I$ part of one of the operators that feature in (3.10) and (3.11). For example, the $C_{4}$ operator transforms the state as

$$
\left|\Psi_{i}\right\rangle \rightarrow e^{-\frac{\pi i}{2} \hat{J}_{3}-\pi i \hat{I}_{1}}\left|\Psi_{i}\right\rangle=N_{i j}\left|\Psi_{j}\right\rangle,
$$

for some matrix $N$. A total wave function,

$$
|\Psi\rangle=\sum_{i} \Phi_{i}\left|\Psi_{i}\right\rangle
$$

will be invariant under (3.10) and (3.11) if the $\Phi_{i}$ transform as

$$
\Phi_{i} \rightarrow e^{\frac{\pi i}{2} \hat{S}_{3}+\pi i \hat{T}_{1}} \Phi_{i} \rightarrow N_{j i}^{-1} \Phi_{j}=N_{i j}^{*} \Phi_{j},
$$

that is, if the $\Phi_{i}$ transform as the dual representation compared to the $\left|\Psi_{i}\right\rangle{ }^{1}$. For the unitary groups, the dual matrix representations are simply complex conjugates of each other.

To construct permissible wave functions, we must first understand the representations of the symmetry groups, which we denote $\mathcal{G}_{J I}$ and $\mathcal{G}_{S T}$. First, we consider $\mathcal{G}_{J I}$, which is generated by the $J / I$ part of the full symmetry transformations (3.10) and (3.11). These are

$$
\begin{aligned}
\hat{C}_{4}^{J, I} & =\exp \left(-\frac{\pi i}{2} \hat{J}_{3}-\pi i \hat{I}_{1}\right) \\
\hat{C}_{3}^{J, I} & =\exp \left(-\frac{2 \pi i}{3 \sqrt{3}}\left(\hat{J}_{1}+\hat{J}_{2}+\hat{J}_{3}\right)-\frac{2 \pi i}{3} \hat{I}_{3}\right) .
\end{aligned}
$$

The group is closely related to the cubic group $O$. The difference can be seen by considering the $C_{4}$ element. Applying this four times gives a $2 \pi$ rotation and a $4 \pi$ isorotation. Hence, the wave function should pick up an overall minus sign under $\left(C_{4}\right)^{4}$, meaning that $\left(C_{4}\right)^{4} \neq e$, where $e$ is the identity operator. Thus, $\mathcal{G}_{J I}$ is not homomorphic to $O$. In contrast, $\left(C_{3}\right)^{3}=e$. Overall, the group has 48 elements, though we neglect the inversion operator for now. The character table of $\mathcal{G}_{J I}$ is displayed in Table I. It contains the character table of $O$ since the quotient of $\mathcal{G}_{J I}$ by $2 \pi$ isorotations is $O$. There are three irreducible representations (irreps) not contained in $O$. A four-dimensional irrep, denoted $\mathbf{4}$, descends from the fundamental representation of

\footnotetext{
${ }^{1}$ We thank Jonathan Rawlinson for bringing this to our attention.
}

TABLE I. The character table for $\mathcal{G}_{J I}$ and $\mathcal{G}_{S T}$. The first five rows show the character table for the usual cubic group.

\begin{tabular}{llrrc}
\hline \hline Irrep & $e$ & $\hat{C}_{4}$ & $\hat{C}_{3}$ & $2 \pi$ rotation \\
\hline $\mathbf{1}^{+}$ & 1 & 1 & 1 & 1 \\
$\mathbf{1}^{-}$ & 1 & -1 & 1 & 1 \\
$\mathbf{2}^{\mathbf{0}}$ & 2 & 0 & -1 & 2 \\
$\mathbf{3}^{+}$ & 3 & 1 & 0 & 3 \\
$\mathbf{3}^{-}$ & 3 & -1 & 0 & 3 \\
$\mathbf{2}^{+}$ & 2 & $\sqrt{2} i$ & -1 & -2 \\
$\mathbf{2}^{-}$ & 2 & $-\sqrt{2} i$ & -1 & -2 \\
$\mathbf{4}$ & 4 & 0 & 1 & -4 \\
\hline \hline
\end{tabular}

$S U(2)_{J} \times S U(2)_{I}$. The remaining irreps both have dimension 2 , and so we label them $\mathbf{2}^{+}$and $\mathbf{2}^{-}$. The symmetry group $\mathcal{G}_{S T}$ is isomorphic to $\mathcal{G}_{J I}$, and hence they share the same character table.

The character table may be used to decompose the set of spin states

$$
\left\{\left|J j_{3} J\right\rangle\left|I i_{3} I\right\rangle \mid j_{3}=-J, \ldots, J, i_{3}=-I, \ldots, I\right\}
$$

into irreducible parts. As an example, take the $J=3 / 2$, $I=1 / 2$ basis. First, we find the matrix representation of the operators (4.4) using $\boldsymbol{M}^{*}$, as explained in Sec. III. This is an eight-dimensional representation that satisfies

$$
\operatorname{Tr}\left(C_{3}^{J=\frac{3}{2}, I=\frac{1}{2}}\right)=-1 \quad \text { and } \quad \operatorname{Tr}\left(C_{4}^{J=\frac{3}{2}, I=\frac{1}{2}}\right)=0 .
$$

Comparing these traces to the character table, one finds that this representation contains a single copy of $\mathbf{4}, \mathbf{2}^{+}$, and $\mathbf{2}^{-}$. One can do a similar decomposition for any $(J, I)$ pair, and the results for a number of different pairs are shown in Table II. The decomposition is the same for the $S / T$ wave functions since the symmetry groups are isomorphic.

Using Table II, we can quickly see which combinations of $J, I, S$, and $T$ give permissible wave functions, and how many exist. A permissible wave function exists if there is a matching irreducible factor between the $J / I$ part and the $S / T$ part. For instance, there is one allowed state with $(J, I, S, T)=(1 / 2,1 / 2,1 / 2,1 / 2)$, since there is one

TABLE II. The decomposition of spin states into irreducible parts.

\begin{tabular}{lc}
\hline \hline$(J, I)$ or $(S, T)$ & Irreducible decomposition \\
\hline$\left(\frac{1}{2}, \frac{1}{2}\right)$ & $\mathbf{4}$ \\
$\left(\frac{1}{2}, \frac{3}{2}\right)$ & $\mathbf{2}^{+} \oplus \mathbf{2}^{-} \oplus \mathbf{4}$ \\
$\left(\frac{3}{2}, \frac{1}{2}\right)$ & $\mathbf{2}^{+} \oplus \mathbf{2}^{-} \oplus \mathbf{4}$ \\
$\left(\frac{5}{2}, \frac{1}{2}\right)$ & $\mathbf{2}^{+} \oplus \mathbf{2}^{-} \oplus \mathbf{4} \oplus \mathbf{4}$ \\
$\left(\frac{7}{2}, \frac{1}{2}\right)$ & $\mathbf{2}^{+} \oplus \mathbf{2}^{-} \oplus \mathbf{4} \oplus \mathbf{4} \oplus \mathbf{4}$ \\
$\left(\frac{3}{2}, \frac{3}{2}\right)$ & $\mathbf{2}^{+} \oplus \mathbf{2}^{-} \oplus \mathbf{4} \oplus \mathbf{4} \oplus \mathbf{4}$ \\
\hline \hline
\end{tabular}


factor of 4 in common for $(J, I)=(1 / 2,1 / 2)$ and $(S, T)=(1 / 2,1 / 2)$. There are four allowed states with $(J, I, S, T)=(3 / 2,1 / 2,5 / 2,1 / 2)$ : two arising from the 4 irreps, one arising from the $\mathbf{2}^{+}$irrep, and one arising from the $\mathbf{2}^{-}$irrep. There is only one allowed state with $(J, I, S, T)=(1 / 2,1 / 2,3 / 2,1 / 2)$. We denote the $i$ th wave function, whose $J / I$ and $S / T$ parts each transform as the irrep $\mathbf{X}$, having spins $(J, I, S, T)$ and space-fixed projection $t_{3}$ as

$$
\left|J I S T ; X_{i} t_{3}^{\prime}\right\rangle .
$$

We omit $i$ if there is only one such state.

To explicitly construct the permissible wave functions, we must choose a concrete realization of the irreps. Here, we make such a choice. The $\mathbf{4}$ irrep descends from the fundamental representation of $S U(2) \times S U(2)$. Hence, an obvious choice of basis is the usual spin states ${ }^{2}$ with spins $(1 / 2,1 / 2)$. The transformations then correspond to the matrices

$$
\begin{aligned}
N_{C_{3}}^{4} & =\frac{1}{\sqrt{2}}\left(\begin{array}{cccc}
e^{\frac{i \pi}{12}} & 0 & e^{-\frac{5 i \pi}{12}} & 0 \\
0 & e^{-\frac{7 i \pi}{12}} & 0 & e^{\frac{11 i \pi}{12}} \\
e^{\frac{i \pi}{12}} & 0 & e^{\frac{7 i \pi}{12}} & 0 \\
0 & e^{-\frac{7 i \pi}{12}} & 0 & e^{-\frac{i \pi}{12}}
\end{array}\right) \\
\text { and } N_{C_{4}}^{4} & =\left(\begin{array}{cccc}
0 & e^{\frac{i \pi}{4}} & 0 & 0 \\
e^{\frac{i \pi}{4}} & 0 & 0 & 0 \\
0 & 0 & 0 & e^{\frac{3 i \pi}{4}} \\
0 & 0 & e^{\frac{3 i \pi}{4}} & 0
\end{array}\right) .
\end{aligned}
$$

The two-dimensional irreps are simpler. For the $\mathbf{2}^{+}$irrep, we may use a basis that transforms as

$$
N_{C_{3}}^{2^{+}}=\frac{1}{\sqrt{2}}\left(\begin{array}{cc}
e^{-\frac{3 i \pi}{4}} & e^{\frac{3 i \pi}{4}} \\
e^{\frac{i \pi}{4}} & e^{\frac{3 i \pi}{4}}
\end{array}\right) \quad \text { and } \quad N_{C_{4}}^{2^{+}}=\frac{1}{\sqrt{2}}\left(\begin{array}{cc}
e^{\frac{3 i \pi}{4}} & 0 \\
0 & e^{\frac{i \pi}{4}}
\end{array}\right) .
$$

Finally, there is a basis for the $\mathbf{2}^{-}$irrep, which transforms as

$N_{C_{3}}^{2^{-}}=\frac{1}{\sqrt{2}}\left(\begin{array}{cc}e^{-\frac{3 i \pi}{4}} & e^{\frac{3 i \pi}{4}} \\ e^{\frac{i \pi}{4}} & e^{\frac{3 i \pi}{4}}\end{array}\right) \quad$ and $\quad N_{C_{4}}^{2^{-}}=\frac{1}{\sqrt{2}}\left(\begin{array}{cc}e^{-\frac{i \pi}{4}} & 0 \\ 0 & e^{-\frac{3 i \pi}{4}}\end{array}\right)$.

We can now construct a wave function that satisfies (3.10) and (3.11), and we do so for $(J, I, S, T)=$ $(1 / 2,1 / 2,1 / 2,1 / 2)$. There is a set of $(J, I)=$ $(1 / 2,1 / 2)$ states that transform as $\mathbf{4}$ and in particular

\footnotetext{
${ }^{2}$ Note that the spin states $\left|J j_{3} J\right\rangle$ and $\left|I i_{3} I\right\rangle$ are not the usual spin states. Their generating matrices are conjugate to the usual ones.
}

transform as (4.8) under the action (4.4). These are given by

$$
\begin{aligned}
\left|\Psi_{i}\right\rangle= & \left(\left|\frac{1}{2} \frac{1}{2} \frac{1}{2}\right\rangle\left|\frac{1}{2}-\frac{1}{2} \frac{1}{2}\right\rangle,-\left|\frac{1}{2} \frac{1}{2} \frac{1}{2}\right\rangle\left|\frac{1}{2} \frac{1}{2} \frac{1}{2}\right\rangle,\right. \\
& \left.-i\left|\frac{1}{2}-\frac{1}{2} \frac{1}{2}\right\rangle\left|\frac{11}{2} \frac{1}{2} \frac{1}{2}\right\rangle, i\left|\frac{1}{2}-\frac{1}{2} \frac{1}{2}\right\rangle\left|\frac{1}{2}-\frac{11}{2} \frac{1}{2}\right\rangle\right)_{i} .
\end{aligned}
$$

The $(S, T)=(1 / 2,1 / 2)$ states that transform as (4.3), where $N$ is given by (4.8), are

$$
\begin{aligned}
\Phi_{i}= & \left(\left|\frac{1}{2} \frac{1}{2} t_{3}^{\prime}\right\rangle\left|\frac{1}{2}-\frac{1}{2}-t_{3}^{\prime}\right\rangle,-\left|\frac{1}{2} \frac{1}{2} t_{3}^{\prime}\right\rangle\left|\frac{1}{2} \frac{1}{2}-t_{3}^{\prime}\right\rangle\right. \\
& \left.i\left|\frac{1}{2}-\frac{1}{2} t_{3}^{\prime}\right\rangle\left|\frac{1}{2} \frac{1}{2}-t_{3}^{\prime}\right\rangle,-i\left|\frac{1}{2}-\frac{1}{2} t_{3}^{\prime}\right\rangle\left|\frac{1}{2}-\frac{1}{2}-t_{3}^{\prime}\right\rangle\right)_{i} .
\end{aligned}
$$

There are two sets of states that are identical apart from their space-fixed projections in $S / T$ space. Note that the bases $\left|\Psi_{i}\right\rangle$ and $\Phi_{i}$ are simply related - their coefficients are complex conjugates. This is true in general since the $S / T$ wave functions must satisfy (4.3). Combining these two bases gives two wave functions with $(J, I, S, T)=$ $(1 / 2,1 / 2,1 / 2,1 / 2)$ that are permitted by the cubic symmetry of the system. They are

$$
\begin{aligned}
\left|\frac{1}{2} \frac{1}{2} \frac{1}{2} \frac{1}{2} ; 4 t_{3}^{\prime}\right\rangle= & \frac{1}{2}\left(\left|\frac{1}{2} \frac{1}{2}\right\rangle\left|\frac{1}{2}-\frac{1}{2}\right\rangle\left|\frac{1}{2} \frac{1}{2} t_{3}^{\prime}\right\rangle\left|\frac{1}{2}-\frac{1}{2}-t_{3}^{\prime}\right\rangle\right. \\
& +\left|\frac{1}{2} \frac{1}{2}\right\rangle\left|\frac{1}{2} \frac{1}{2}\right\rangle\left|\frac{1}{2} \frac{1}{2} t_{3}^{\prime}\right\rangle\left|\frac{1}{2} \frac{1}{2}-t_{3}^{\prime}\right\rangle \\
& +\left|\frac{1}{2}-\frac{1}{2}\right\rangle\left|\frac{1}{2} \frac{1}{2}\right\rangle\left|\frac{1}{2}-\frac{1}{2} t_{3}^{\prime}\right\rangle\left|\frac{1}{2} \frac{1}{2}-t_{3}^{\prime}\right\rangle \\
& \left.+\left|\frac{1}{2}-\frac{1}{2}\right\rangle\left|\frac{1}{2}-\frac{1}{2}\right\rangle\left|\frac{1}{2}-\frac{1}{2} t_{3}^{\prime}\right\rangle\left|\frac{1}{2}-\frac{1}{2} t_{3}^{\prime}\right\rangle\right),
\end{aligned}
$$

where we have suppressed the space-fixed projections of $J$ and $I$ for economy. To find wave functions with larger values of $J, I, S$, and $T$, we can simply repeat the process. The wave functions are large, complicated objects, as one would expect from quantization of an 11-dimensional system. We tabulate the bases of spin states that transform as (4.8), (4.9), and (4.10) in Appendix A. These can be used to construct wave functions with high spins.

\section{PARITY AND ADDITIONAL SYMMETRIES}

\section{A. Parity}

In the Skyrme model, the inversion operator is easily expressed in terms of the pion field, $\boldsymbol{\pi}(\boldsymbol{x})$. It is 


$$
\hat{P}: \boldsymbol{\pi}(\boldsymbol{x}) \rightarrow-\pi(-x)
$$

This inverts the orientation of the Skyrmion in space and in isospace. To apply the operator to our states, we must first express it in terms of the coordinates on the configuration space. We can split this into two parts, one acting on the overall spin and isospin space $\hat{P}_{J, I}$ and another acting on the relative space $\hat{P}_{S, T}$. For our setup, $\hat{P}_{J, I}$ is easier to express using a momentum operator,

$$
\hat{P}_{J, I}=\exp \left(-i \hat{I}_{3} \pi\right)
$$

while the relative part is simplest to describe using an explicit coordinate transformation,

$\hat{P}_{S, T}:(\theta, \phi, \alpha, \beta, \gamma) \rightarrow(\pi-\theta, \phi+\pi, \alpha+\pi, \beta+\pi, \pi-\gamma)$.

The equivalence between (5.1) and the combined action of (5.2) and (5.3) is shown in Fig. 4, at a generic point in configuration space.

Using properties of the Wigner functions, we find that the inversion operator acts on the basis states as

$$
\begin{aligned}
& \hat{P}\left|J j_{3}\right\rangle\left|I i_{3}\right\rangle\left|S s_{3}-t_{3}^{\prime}\right\rangle\left|T t_{3} t_{3}^{\prime}\right\rangle \\
& \quad=(-1)^{1+S+T+t_{3}-i_{3}}\left|J j_{3}\right\rangle\left|I i_{3}\right\rangle\left|S s_{3} t_{3}^{\prime}\right\rangle\left|T t_{3}-t_{3}^{\prime}\right\rangle .
\end{aligned}
$$

This can be used to construct states with definite parity $P$ that satisfy $\hat{P}|\Psi\rangle=P|\Psi\rangle= \pm|\Psi\rangle$. To find definite parity wave functions, we begin with a set of degenerate energy eigenstates. We have not yet constructed such states but will do so soon. There are only two states with $J=I=S=T=1 / 2$, degenerate in their $t_{3}^{\prime}$ value. The space-fixed projection cannot affect the energy, and so these states, displayed in (4.15), must be energy eigenstates and can be used as a basis for the definite parity states. The definite parity states are
$|P= \pm 1\rangle=\frac{1}{\sqrt{2}}\left(\left|\frac{1}{2} \frac{1}{2} \frac{1}{2} \frac{1}{2} ; 4 \frac{1}{2}\right\rangle \pm\left|\frac{1}{2} \frac{1}{2} \frac{1}{2} \frac{1}{2} ; 4-\frac{1}{2}\right\rangle\right)$.

There is an alternative way to calculate the parity at certain points in configuration space-those in which the Skyrme configuration has a reflection symmetry. At these, $\hat{P}$ can be written in terms of the $\hat{J}$ and $\hat{I}$ momentum operators. This gives a nontrivial consistency check on the global parity operator. As an example, consider the configuration displayed in Fig. 5(a). This is the point $\boldsymbol{\alpha}_{z}=(\theta, \phi, \alpha, \beta, \gamma)=(\pi / 2, \pi / 4,0, \pi / 2,0)$. At this point, the parity operator is

$$
\left.\hat{P}\right|_{\alpha_{z}}=\exp \left(-i \pi \hat{J}_{3}\right) \exp \left(i \pi \hat{I}_{3}\right) .
$$

We may apply this operator to the wave function evaluated at $\boldsymbol{\alpha}_{z}$ and should obtain the same parity as before. For the negative-parity wave function in (5.5), we find that

$$
\begin{aligned}
\left.\left.\hat{P}\right|_{\boldsymbol{\alpha}_{z}}|P=-1\rangle\right|_{\boldsymbol{\alpha}_{z}}= & \frac{1}{8 \sqrt{2}} e^{-i \pi \hat{J}_{3}+i \pi \hat{I}_{3}}\left(e^{\frac{i \pi}{8}}\left|\frac{1}{2} \frac{1}{2}\right\rangle\left|\frac{1}{2}-\frac{1}{2}\right\rangle\right. \\
& \left.+e^{-\frac{i \pi}{8}}\left|\frac{1}{2}-\frac{1}{2}\right\rangle\left|\frac{1}{2} \frac{1}{2}\right\rangle\right) \\
= & -\left.|P=-1\rangle\right|_{\boldsymbol{\alpha}_{z}},
\end{aligned}
$$

as expected. If the global parity operator is particularly difficult to write down, one could use this local method to construct definite parity states. For large spins, one would need to evaluate the wave function at several different points in configuration space where the configuration has a reflection symmetry. More of these are displayed in Fig. 5.

Each energy eigenstate (which will be constructed in Sec. VI) has a $2 n$ degeneracy in which

$$
2 n=\min (2 S+1,2 T+1) .
$$

Half of these have positive parity, and other half have negative parity. Hence, there is at least parity doubling for each state. This is not seen in the ${ }^{5} \mathrm{He} /{ }^{5} \mathrm{Li}$ energy spectra. The doubling is ultimately due to the lack of symmetry in our configuration space. The configurations with the largest

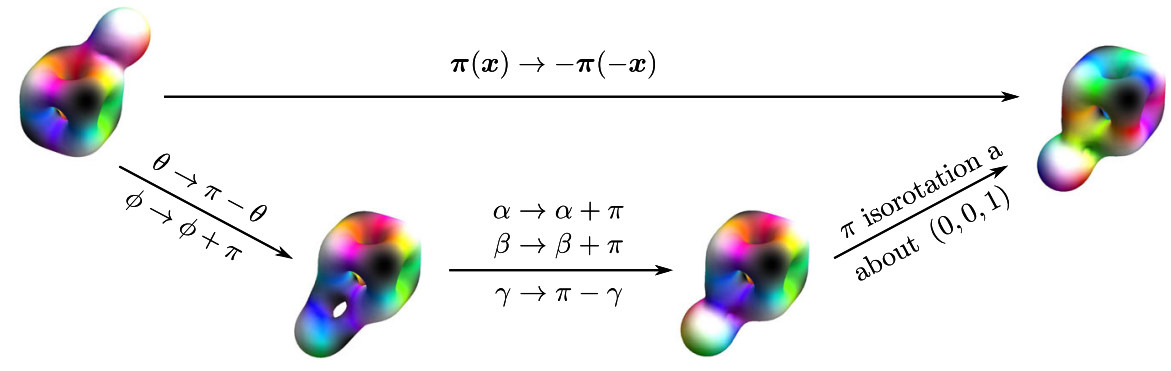

FIG. 4. A visual representation of the equivalence between the inversion operator acting on the Skyrme field and its realization in our configuration space. 
(a)

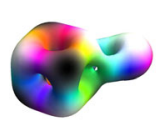

(b)

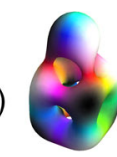

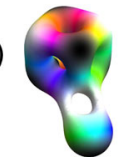

(d)

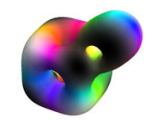

FIG. 5. Four points in the configuration space with a reflection symmetry. The $B=1$ lies at an edge, a face, a corner, and a different edge of the cube in $(a),(b),(c)$, and $(d)$, respectively.

symmetry group include those seen in Figs. 5(a) and 5(d), which have an overall $C_{2}$ symmetry. This order-2 group partitions the total spin/isospin basis into two sets: those that transform trivially under the $C_{2}$ element and those that pick up a sign. One of these sets is disregarded due to the FR constraints. The remaining, allowed, wave functions are once again partitioned in two by the order-2 parity operator. For half-integer spin and isospin, the set of free states is divisible by 4 , and so each positive-parity state has a negative-parity partner. For example, there are four $J=$ $I=1 / 2$ basis states. Two are allowed by the FR constraints: one has positive parity, and the other has negative parity. By this reasoning, if a Skyrmion has only $C_{2}$ symmetry, its energy spectrum will contain approximate parity doubling, ${ }^{3}$ almost never seen in experimental data. This appears to be a problem for Skyrme models with low classical binding energy. For example, the lightly bound model [12] contains parity doubling for many of its Skyrmions. This argument suggests that one should attempt to construct a Skyrme model with low classical binding energy and high symmetry. There have been several attempts to write down such a model. One is to include vector mesons in the Lagrangian. Recently, it was shown that inclusion of the rho meson reduces the classical binding energy by $2 / 3$, while retaining the symmetry group of each Skyrmion up to baryon number 8 [22]. If one integrates out the mesons, a term that contains sixth-order derivatives of the pion field appears [23]. If one only includes this term, the theory is Bogomol'nyi-PrasadSommerfield (BPS) (i.e., has no classical binding energy), and so, unsurprisingly, its inclusion lowers the binding energy [4]. Numerical work [11] has shown that the classical symmetries do remain for a family of Lagrangians in which the sixth-order term does not completely dominate. One can also modify the pion potential term that, again, has been shown to reduce binding energies while retaining much of the Skyrmion symmetries [8-10]. In all of these models, quantum effects arising from the spin of the $B=1$ Skyrmion spoil the small classical binding energies. An alternative approach is to start with a classically tightly bound but highly symmetric Skyrme model and reduce the binding energy by taking account of more modes in the quantization procedure, as

\footnotetext{
${ }^{3}$ The structure of the moment of inertia tensor may break the degeneracy slightly.
}

suggested in Refs. [7,24]. If the classical $B=5$ Skyrmion has a symmetry group of order 4 , the parity doubling problem is avoided.

Finally, we compare our results about parity with the shell model, which does not contain doubling. The key difference is that, in the shell model, the core and additional particle are individual quantum objects. They are then combined to make a two-cluster system. Since the core is already quantized, it has spherical symmetry, and the additional particle is governed by a central potential. In our case, the additional particle feels the classical structure of the core, which has much less symmetry than the spherical core of the shell model. This reduction in symmetry means that relatively more states are allowed in our model, leading to incorrect results. Our clusters are combined at the classical level and then quantized. We could perhaps reproduce the shell model results by quantizing the $B=4$ and $B=1$ separately and then combining them. It may be of interest to compare these two methods and find out when each is appropriate.

\section{B. $C_{2}$ symmetry}

The configuration space appears to contain more symmetries than we have considered so far. Here, we will consider one such symmetry and explain why it is in fact included in our calculation.

Consider the point in Fig. 5(a), $\boldsymbol{\alpha}_{z}$. This has a $C_{2}$ symmetry, realized as

$$
\left.\exp \left(-\frac{\pi i}{\sqrt{2}}\left(\hat{J}_{1}+\hat{J}_{2}\right)-\pi i \hat{I}_{1}\right)|\Psi\rangle\right|_{\boldsymbol{\alpha}_{z}}=\left.|\Psi\rangle\right|_{\alpha_{z}}
$$

The cubic group that we considered earlier includes a closely related $C_{2}$ element,

$\exp \left(-\frac{\pi i}{\sqrt{2}}\left(\hat{J}_{1}+\hat{J}_{2}\right)-\pi i \hat{I}_{1}+\frac{\pi i}{\sqrt{2}}\left(\hat{S}_{1}+\hat{S}_{2}\right)+\pi i \hat{T}_{1}\right)|\Psi\rangle=|\Psi\rangle$

The $S / T$ part of this transformation can be viewed as a coordinate transformation on the $S / T$ space. It takes $\boldsymbol{\alpha}_{z}$ to itself. Hence,

$$
\left.\left(\frac{\pi i}{\sqrt{2}}\left(\hat{S}_{1}+\hat{S}_{2}\right)+\pi i \hat{T}_{1}\right)|\Psi\rangle\right|_{\alpha_{z}}=\left.|\Psi\rangle\right|_{\alpha_{z}}
$$

meaning that the $S / T$ part of (5.11) is trivial at the point $\boldsymbol{\alpha}_{z}$ and thus (5.10) is satisfied.

Provided that the constraint (5.10) is satisfied, several other constraints are automatically dealt with. Denote the point in Fig. 5(d) as $\boldsymbol{\alpha}_{\boldsymbol{y}}=(\pi / 4,0,4 \pi / 3, \pi / 2, \pi / 2)$. This should satisfy the constraint 


$$
\begin{aligned}
\left.\hat{C}_{2}^{y}|\Psi\rangle\right|_{\alpha_{y}} \equiv & \exp \left(-\frac{\pi i}{\sqrt{2}}\left(\hat{J}_{1}+\hat{J}_{3}\right)-\pi i\left(\cos (4 \pi / 3) \hat{I}_{1}\right.\right. \\
& \left.\left.+\sin (4 \pi / 3) \hat{I}_{2}\right)\right)\left.|\Psi\rangle\right|_{\boldsymbol{\alpha}_{y}}=\left.|\Psi\rangle\right|_{\boldsymbol{\alpha}_{y}}
\end{aligned}
$$

To show that this does hold, we will need to introduce some extra terminology. Denote the $C_{3}$ operator that relates the configurations in Figs. 5(a) and 5(d) in $S / T$ space as

$$
\hat{C}_{3}^{S, T}=\exp \left(\frac{2 \pi i}{3 \sqrt{3}}\left(\hat{S}_{1}+\hat{S}_{2}+\hat{S}_{3}\right)+\frac{2 \pi i}{3} \hat{T}_{3}\right) .
$$

The total wave function has been constructed to be invariant under (5.14) combined with the related action on the $J / I$ space:

$$
\hat{C}_{3}^{J, I}=\exp \left(-\frac{2 \pi i}{3 \sqrt{3}}\left(\hat{J}_{1}+\hat{J}_{2}+\hat{J}_{3}\right)-\frac{2 \pi i}{3} \hat{I}_{3}\right) .
$$

Hence, the total wave function satisfies

$$
\hat{C}_{3}^{S, T} \hat{C}_{3}^{J, I}|\Psi\rangle=|\Psi\rangle
$$

which means that

$$
\left.|\Psi\rangle\right|_{\boldsymbol{\alpha}_{z}}=\left.\left(\hat{C}_{3}^{S, T}\right)^{-1}|\Psi\rangle\right|_{\alpha_{y}}=\left.\hat{C}_{3}^{J, I}|\Psi\rangle\right|_{\alpha_{y}} .
$$

The first equality is simply a coordinate transformation on the $S / T$ space, while the second is a consequence of (5.16).

Now, we can consider the wave function at $\boldsymbol{\alpha}_{\boldsymbol{y}}$. It satisfies

$$
\begin{aligned}
\left.\hat{C}_{2}^{y}|\Psi\rangle\right|_{\alpha_{y}} & =\left(\hat{C}_{3}^{J, I}\right)^{-1}\left(\hat{C}_{3}^{J, I} \hat{C}_{2}^{y}\left(\hat{C}_{3}^{J, I}\right)^{-1}\right)\left(\left.\hat{C}_{3}^{J, I}|\Psi\rangle\right|_{\alpha_{y}}\right) \\
& =\left.\left(\hat{C}_{3}^{J, I}\right)^{-1} \hat{C}_{2}^{z}|\Psi\rangle\right|_{\alpha_{z}} \\
& =\left.\left(\hat{C}_{3}^{J, I}\right)^{-1}|\Psi\rangle\right|_{\alpha_{z}} \\
& =|\Psi\rangle_{\alpha_{y}}
\end{aligned}
$$

Hence, the wave function obeys the constraint at $\boldsymbol{\alpha}_{y}$, provided it also obeys the constraint at $\boldsymbol{\alpha}_{z}$, due to the group structure. This type of argument is rather straightforward, but it contains a key message: get the symmetry of the configuration space correct, and everything else will follow.

\section{Enhanced $D_{2}$ symmetry}

There is an additional symmetry we may consider. This exists just outside of our configuration space. Consider the point $\boldsymbol{\alpha}_{z}$ in Fig. 5(a). In the standard Skyrme model, when the $B=1$ Skyrmion is brought closer to the cube, the configuration deforms into the well-known $D_{2}$-symmetric $B=5$ Skyrmion. This deformation process is displayed in Fig. 6 . The $B=5$ Skyrmion has $D_{2}$ symmetry, generated by two elements. The first is the $C_{2}$ symmetry (5.11), and the other is another $C_{2}$ element that enforces an extra constraint on the wave function at the point $\boldsymbol{\alpha}_{z}$,

$$
\begin{aligned}
& \left.\exp \left(-i \pi\left(\frac{1}{2} \hat{J}_{1}-\frac{1}{2} \hat{J}_{2}+\frac{1}{\sqrt{2}} \hat{J}_{3}\right)-i \pi\left(\frac{1}{\sqrt{2}} \hat{I}_{2}-\frac{1}{\sqrt{2}} \hat{I}_{3}\right)\right)|\Psi\rangle\right|_{\boldsymbol{\alpha}_{z}} \\
& =-\left.|\Psi\rangle\right|_{\boldsymbol{\alpha}_{z}} .
\end{aligned}
$$

We calculated the FR sign using the approach of Krusch based on the rational map approximation [18]. We can check if our definite parity energy eigenstates obey the constraints by evaluating the wave functions at $\boldsymbol{\alpha}_{z}$. When there are degenerate eigenstates $(S, T>1 / 2)$, we can attempt to construct linear combinations of the states that obey the constraints. If the wave function satisfies the constraint at $\boldsymbol{\alpha}_{z}$, it is automatically enforced at the other $D_{2}$-symmetric points due to the argument used in the previous subsection.

We do not rule out states that do not satisfy (5.19) since our configuration space does not explicitly include the $D_{2}$ symmetric Skyrmions. However, their existence still affects the energies of our states. To see why, consider a quantization that includes a parameter $\tau$ describing the flow seen in Fig. 6 (it could be thought of as the radial coordinate), and suppose the $D_{2}$ symmetry is restored at $\tau^{*}$. The wave functions of this system will look schematically like

$$
u(\tau) \times|\Psi\rangle,
$$

where the $|\Psi\rangle$ are the wave functions we have calculated in this paper. Now, consider a "rotational" state that is allowed by the $D_{2}$ symmetry, denoted $|\Psi ; D\rangle$. Here, the wave function $u(\tau)$ coupled to $|\Psi ; D\rangle$ is not restricted in any way. Now, consider a rotational wave function not allowed by the $D_{2}$ symmetry (5.19), denoted $|\Psi ; C\rangle$. If this is coupled to a wave function $u(\tau)$, then the only way to satisfy the $D_{2}$ symmetry (5.19) is for $u(\tau)$ to vanish at $\tau^{*}$. This imposes an extra constraint on the wave function.

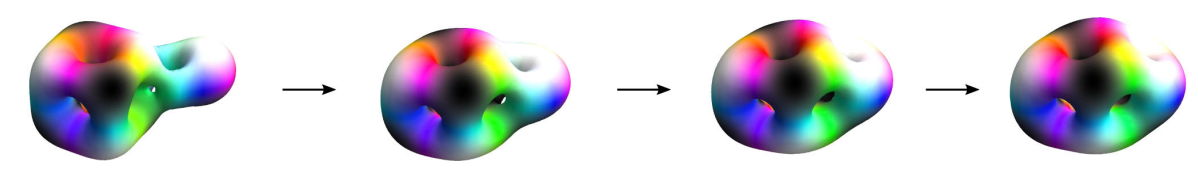

FIG. 6. Gradient flow from the configuration in Fig. 5(a) to the $D_{2}$-symmetric Skyrmion. This process is carried out in the standard Skyrme model with dimensionless pion mass $m_{1}=1$. 
Constrained wave functions naturally have higher energy, and so the total wave function containing $|\Psi ; C\rangle$ has more energy than the one containing $|\Psi ; D\rangle$. The implication for our model is that wave functions not allowed by (5.19) should gain an additional energy contribution. We call this the constraint energy. If one thinks of $\tau$ as a radial coordinate, the $|\Psi ; D\rangle$ states are coupled to a ground-state radial wave function, while the $|\Psi ; C\rangle$ states would be coupled to an excited radial wave function, since it must vanish at the minimum of the potential energy (for the standard Skyrme model).

The size of the constraint energy is hard to measure. To do so properly, one should consider a configuration space and corresponding quantization scheme that includes a coordinate like $\tau$. Even to estimate the contribution, one needs to understand the distance between the configurations we consider in our model and the $D_{2}$-symmetric Skyrmion. This depends crucially on the interpretation of our configuration space $\mathcal{M}$, briefly mentioned in Sec. II. One may interpret $\mathcal{M}$ as already including the $D_{2}$ Skyrmion at the point $\boldsymbol{\alpha}_{z}$. The relative coordinates between the clusters are then internal, vibrational excitations of the $D_{2}$-symmetric $B=5$ Skyrmion. The two-cluster picture is then just a convenient way to think about these coordinates. In this picture, the constraint energy is very large. To see if this interpretation is possible, one would need to do a small amplitude analysis of the $B=5$ Skyrmion to see if there is a map between its vibrational d.o.f. and those in this model. The true picture is likely somewhere between this interpretation and the interpretation of the Skyrmion as two weakly bound clusters. To understand it properly, a more thorough investigation of the $B=5$ configuration space is required.

\section{CALCULATING ENERGIES}

\section{A. Kinetic energy}

The Hamiltonian $\mathcal{H}$ of the system is simple to express in terms of the body-fixed momenta of the individual Skyrmions. Ignoring deformations caused by the Skyrmion interaction that will change the moment of inertia tensors, the kinetic part of the energy is

$$
\begin{aligned}
\mathcal{H}_{\text {free }}= & \frac{1}{2}\left(\boldsymbol{L}^{(4) T} V_{4}^{-1} \boldsymbol{L}^{(4)}+\boldsymbol{K}^{(4) T} U_{4}^{-1} \boldsymbol{K}^{(4)}+\boldsymbol{L}^{(1) T} V_{1}^{-1} \boldsymbol{L}^{(1)}\right. \\
& \left.+\left(M_{1} R^{2}\right)^{-1} \boldsymbol{l}^{T} \boldsymbol{l}\right)
\end{aligned}
$$

where $V_{i}$ and $U_{i}$ are the moment of inertia tensors associated with the angular and isoangular motion of the $B=i$ Skyrmion, $M_{1}$ is the mass of the $B=1$ Skyrmion, and $R=$ $|\boldsymbol{R}|$ is the (fixed) distance between the Skyrmions. We can express the Hamiltonian in terms of the new momenta by inverting Eqs. (2.5), (2.6), and (2.8). By doing this, we find that

$$
\left(\begin{array}{c}
\boldsymbol{L}^{(4)} \\
\boldsymbol{K}^{(4)} \\
\boldsymbol{L}^{(1)} \\
\boldsymbol{l}
\end{array}\right)=\left(\begin{array}{cccc}
\mathbf{1} & 0 & -\mathbf{1} & 0 \\
0 & \mathbf{1} & 0 & -\mathbf{1} \\
0 & 0 & 0 & -B^{(1) T} B^{(4)} \\
0 & 0 & A^{(4)} & A^{(1)} B^{(1) T} B^{(4)}
\end{array}\right)\left(\begin{array}{c}
\boldsymbol{J} \\
\boldsymbol{I} \\
\boldsymbol{S} \\
\boldsymbol{T}
\end{array}\right)
$$

where 1 represents the $3 \times 3$ unit matrix. The Hamiltonian becomes

$$
\begin{aligned}
\mathcal{H}_{\text {free }} & =\frac{1}{2}\left(\begin{array}{llll}
\boldsymbol{J} & \boldsymbol{I} & \boldsymbol{S} & \boldsymbol{T}
\end{array}\right) \mathcal{G}\left(\begin{array}{l}
\boldsymbol{J} \\
\boldsymbol{I} \\
\boldsymbol{S} \\
\boldsymbol{T}
\end{array}\right), \\
\mathcal{G} & \equiv\left(\begin{array}{cccc}
V_{4}^{-1} & 0 & -V_{4}^{-1} & 0 \\
0 & U_{4}^{-1} & 0 & -U_{4}^{-1} \\
-V_{4}^{-1} & 0 & V_{4}^{-1}+\left(M_{1} R^{2}\right)^{-1} & -\left(M_{1} R^{2}\right)^{-1} A^{(4) T} A^{(1)} B^{(1) T} B^{(4)} \\
0 & -U_{4}^{-1} & -\left(M_{1} R^{2}\right)^{-1} A^{(4) T} A^{(1)} B^{(1) T} B^{(4)} & U_{4}^{-1}+V_{1}^{-1}+\left(M_{1} R^{2}\right)^{-1}
\end{array}\right) .
\end{aligned}
$$

The only complicated term in this expression is the $S / T$ cross term. We are unsure how to evaluate this term, and so we replace it by its expectation value

$$
\begin{aligned}
& \left(M_{1} R^{2}\right)^{-1} A^{(4) T} A^{(1)} B^{(1) T} B^{(4)} \\
& \quad \rightarrow\left(M_{1} R^{2}\right)^{-1}\left\langle A^{(4) T} A^{(1)} B^{(1) T} B^{(4)}\right\rangle=0 .
\end{aligned}
$$

Although we would ideally evaluate this term properly, we are comforted by the fact that $M_{1} R^{2}$ is the largest scale in the metric.
The moment of inertia tensors are all diagonal, and most are proportional to the unit matrix. We use small letters to describe the diagonal elements so that

$$
V_{4}=v_{4} \mathbf{1}, \quad V_{1}=v_{1} \mathbf{1} \quad \text { and } \quad R^{2} M_{1}=\mu \mathbf{1} .
$$

The $B=4$ isospin tensor is slightly more complicated. There are two independent diagonal components. In the orientation we have used, 


$$
\left(U_{4}\right)_{11}=\left(U_{4}\right)_{22}=u_{11} \quad \text { and } \quad\left(U_{4}\right)_{33}=u_{33} .
$$

We fix the free moments of inertia and masses numerically using the results from the standard Skyrme model with dimensionless pion mass $m_{1}=1$. In Skyrme units, this gives

$$
\begin{aligned}
& v_{4}=661, \quad u_{11}=147, \quad u_{33}=176, \\
& M_{4}=613, \quad v_{1}=48 \text { and } M_{1}=168 \text {. }
\end{aligned}
$$

We are left to fit $R$ and hence find $\mu$. We take $R=3$, and this length is also used when we numerically generate the interaction potential.

To find the energy eigenstates, we simply diagonalize the Hamiltonian matrix for each set of allowed states with a fixed set of spins. We review the low-lying states here, and an extensive table of higher-energy states can be found in Appendix B. We list many more states than are experimentally seen. This is not because we are directly interested in them. Rather, we are interested in how their existence affects the lowest-energy states, once the potential is turned on.

The ground state of the free system is the state calculated in (4.15)

$$
\left|\frac{1}{2} \frac{1}{2} \frac{1}{2} \frac{1}{2} ; \mathbf{4} t_{3}^{\prime}\right\rangle
$$

This has energy

$$
E=\frac{3}{8 v_{1}}+\frac{3}{4 \mu} .
$$

The state is doubly degenerate, due to the free $t_{3}^{\prime}$ label. There is one state with negative parity, permitted by the $D_{2}$-symmetric configuration, and one with positive parity, which is not. This state is simple to interpret: since there is no $V^{(4)}$ or $U^{(4)}$ dependence, the $B=4$ core is at rest, while the orbiting nucleon has spin $1 / 2$. Not all states have such a simple interpretation.

Just above the ground state, there is a spin-3/2 state,

$\sqrt{2}\left|\frac{3}{2} \frac{1}{2} \frac{3}{2} \frac{1}{2} ; \mathbf{4} t_{3}^{\prime}\right\rangle+\left|\frac{3}{2} \frac{1}{2} \frac{3}{2} \frac{1}{2} ; \mathbf{2}^{+} t_{3}^{\prime}\right\rangle+\left|\frac{3}{2} \frac{1}{2} \frac{3}{2} \frac{1}{2} ; \mathbf{2}^{-} t_{3}^{\prime}\right\rangle$,

which has energy

$$
E=\frac{3}{8 v_{1}}+\frac{9}{4 \mu} .
$$

Once again, this is doubly degenerate with a negative-parity state, permitted by the $D_{2}$ Skyrmion, and a positive-parity state which is not. Of the four states discussed so far, the two negative-parity states are identified with the two lowenergy states of the ${ }^{5} \mathrm{He} /{ }^{5} \mathrm{Li}$ isodoublet. Experimentally, there are no low-energy positive-parity states. Hence, to match data, the constraint energy from the $D_{2}$-symmetric Skyrmion must be large. This provides some evidence that the correct Skyrme Lagrangian should contain a lowenergy $D_{2}$-symmetric $B=5$ Skyrmion. This is not true for lightly bound models such as Refs. [8-10,12]. It is unclear what happens near the BPS limit of the sextic model [11].

The experimental data then have a large gap of around $15 \mathrm{MeV}$. Our spectrum also has a large gap. The next six states are energetically degenerate. They are

$$
\begin{gathered}
\left|\frac{3}{2} \frac{1}{2} \frac{1}{2} \frac{1}{2} ; \mathbf{4} t_{3}^{\prime}\right\rangle, \\
\left|\frac{5}{2} \frac{1}{2} \frac{1}{2} \frac{1}{2} ; \mathbf{4}_{1} t_{3}^{\prime}\right\rangle, \\
\text { and } \quad\left|\frac{3}{2} \frac{3}{2} \frac{1}{2} \frac{1}{2} ; \mathbf{4}_{2} t_{3}^{\prime}\right\rangle-\sqrt{3}\left|\frac{3}{2} \frac{3}{2} \frac{1}{2} \frac{1}{2} ; \mathbf{4}_{3} t_{3}^{\prime}\right\rangle,
\end{gathered}
$$

where $t_{3}^{\prime}= \pm \frac{1}{2}$. Each of these has a positive- and negativeparity version. They all have energy

$$
E=\frac{3}{8 v_{1}}+\frac{1}{2 u_{11}}+\frac{1}{2 u_{33}}+\frac{3}{v_{4}}+\frac{3}{4 \mu} .
$$

The large degeneracy in the spectrum may be expected since the model is a free theory with a simple kinetic operator. In the full model, the Skyrmions interact, which alters the moment of inertia tensor. This will likely break the degeneracy of these states.

The energy spectrum then becomes rather dense-there are many states with similar energies. Many of these can be found in Appendix B, and we plot their spectrum in Fig. 7. The main success of the free theory is the large energy gap in the spectrum.

\section{B. Potential energy}

Having found the wave functions for the free system, we can now estimate the potential energy contribution. To find the potential $V(\theta, \phi, \alpha, \beta, \gamma)$, we insert a symmetrized product ansatz of a $B=1$ and $B=4$ Skyrmion into the static Skyrme Lagrangian. Numerically, we discretize the angles with a lattice spacing of $\pi / 12$. This amounts to finding $V$ at approximately 2 million points.

The full Hamiltonian $\mathcal{H}$ is the kinetic operator (6.3) plus the numerically generated potential. Denoting our free wave functions as $\left|\Psi^{i}\right\rangle$, the energy spectrum is found by diagonalizing

$$
\left\langle\Psi^{i}|V(\theta, \phi, \alpha, \beta, \gamma)| \Psi^{j}\right\rangle .
$$

Although the wave functions depend on 11 coordinates, the part of the matrix element that depends on $J$ and $I$ is rather 


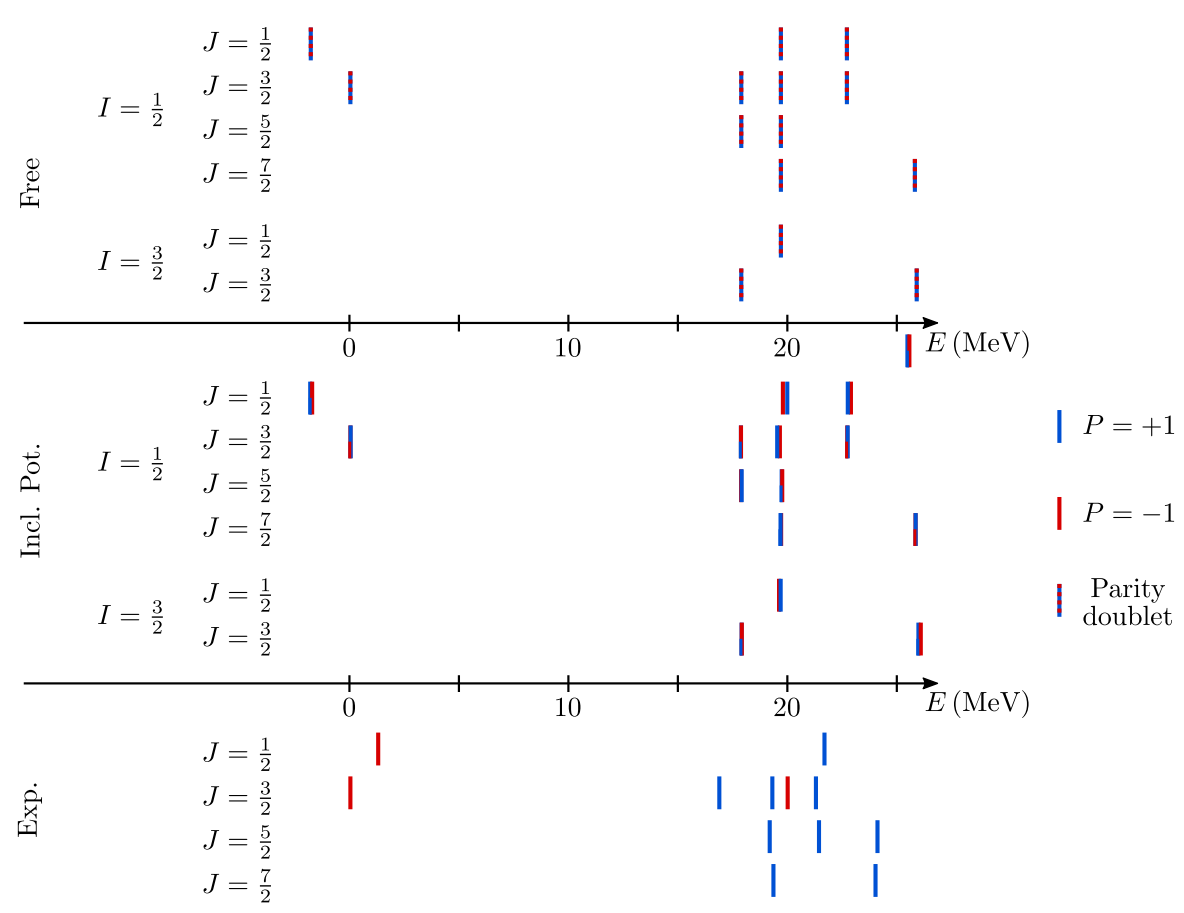

FIG. 7. The energy spectrum for the free theory and after the inclusion of the potential term, compared to the energy spectrum of ${ }^{5} \mathrm{He}$, taken from Ref. [25]. Bars that are red (blue) represent states with negative (positive) parity, while a striped bar represents a parity doublet-two degenerate states with opposite parities.

trivial. Hence, to calculate the matrix element, we only need to do an integration over the five-dimensional $S / T$ space. States with different spins, isospins, and parities have zero mixing, and so we focus on one sector at a time. We only calculate a finite number of free energy eigenfunctions, and so we diagonalize with respect to a truncated basis. We include all states that we found up to and including an energy cutoff, which is

$$
E=\frac{15}{8 v_{1}}+\frac{5}{2 u_{11}}+\frac{1}{2 u_{33}}+\frac{3}{v_{4}}+\frac{15}{4 \mu} .
$$

This means that, for example, we include 14 states with $(J, I)=(1 / 2,1 / 2)$ and 27 states with $(J, I)=(3 / 2,1 / 2)$. We include fewer basis states when considering larger spins. Fortunately, we are primarily interested in the two low-spin cases, in which we have already calculated a large basis of states.

The results of the calculation are shown in Fig. 7 alongside the free energy spectrum and the experimental data, which are taken from Ref. [25]. To calibrate the model, we must choose energy and length scales or, equivalently, choose an energy scale and the value of $\hbar$ in Skyrme units. We take $\hbar=21.26$, the same value taken in Ref. [3]. We use an energy scale in which one Skyrme energy unit is $4.04 \mathrm{MeV}$; this is $70 \%$ smaller than the scale used in Ref. [3]. Our calibration is different since we are using a different quantization scheme. However, it is encouraging that the two sets of parameters are reasonably similar.
The results contain some successes-all experimental states are seen, and the spectrum contains a large gap. There are more free spin- $3 / 2$ states than spin- $1 / 2$ states, and so one may expect, with a larger basis to mix, the low-lying spin-3/2 and $-1 / 2$ states would reverse their order. However, due to the details of the mixing, the spin- $1 / 2$ states remain the lowest-energy ones. Hence, we do not get the correct ground-state spin. The potential only has a small effect on the spectrum since the Skyrmions interact weakly where they are positioned. If we artificially increase the size of the potential, the states remain in the incorrect order. In fact, the size of the gap between the low-energy spin- $1 / 2$ and $-3 / 2$ states increases. This is the opposite of what one would expect from spin-orbit coupling. Hence, the structure of the pion field may not account of the spin-orbit effect in the Skyrme model, at least at weak coupling. We calculated the potential for the Skyrme model with $m_{2}=0$ and $m_{2}=2.8$. The change of potential had little impact on the results.

The model also contains approximate parity doublingnot seen in the ${ }^{5} \mathrm{He} /{ }^{5} \mathrm{Li}$ spectra. We have already suggested one way to remedy the problem: by including the $D_{2}$ symmetric Skyrmion in the configuration space, or energetically punishing the states that are not permitted by this symmetry. This would add a constraint energy contribution to both the low-lying $3 / 2^{+}$and $1 / 2^{+}$states, which are not permitted by this symmetry. In addition, we fail to obtain the $1 / 2^{+}$ground state of the isospin-3/2 nuclei, hydrogen-5, and beryllium-5, although there is a low-energy $1 / 2^{+}$state in our spectrum. These nuclei are highly unstable, so our bound 
two-cluster model is likely unsuitable to accurately describe such states.

\section{FURTHER WORK}

The framework developed in this paper may be applied to a wide range of systems. For any strongly bound Skyrmion with baryon number $B$, our approach can be used to study the $B+1$ nucleus. In the Skyrme model, like many nuclear models, nuclei with $B=4 N$ are particularly stable. For example, the $B=32$ Skyrmion is strongly bound and has cubic symmetry [26]. Hence, the $B=33$ Skyrmion's configuration space will likely have a low-energy subspace that looks like a $32+1$ two-cluster system. This will model certain states of the sulfur-33/chlorine-33 isodoublet. Experimentally, the first two states of these nuclei have spin parity $3 / 2^{+}$and $1 / 2^{+}$, respectively. Applying the free model developed in this paper (which is rather naive), we would find these low-lying states, alongside their negativeparity partners. A more careful study may explain why the positive-parity states are preferred.

There is evidence of large-radius "Hoyle-like" states in the carbon-13 spectrum [27]. In the Skyrme model, these would be described by a single nucleon orbiting the $B=12$ chain-like Skyrmion, which models the Hoyle state. One could describe these novel states using the framework from this paper, but rather than restricting the $B=1$ to a sphere, it should be restricted to an ellipse. In fact, one could restrict the $B=1$ to any surface that reflects the symmetry of the core Skyrmion, such as those with tetrahedral, octahedral, or dodecahedral symmetry. This technique could be used to study nuclei with one nucleon more than the "magic" tetrahedral Skyrmions discussed recently in Refs. [28,29]. One could even repeat the calculation in this paper but insist that the $B=1$ is restricted to a cube so that it is always just touching the $B=4$ core. Then, the Wigner functions in the $S / T$ space used as a basis for the wave functions would be replaced with free wave functions on the surface of the cube.

In the lightly bound Skyrme model [11,12], large Skyrmions are approximately described by a set of individual $B=1$ Skyrmions that take positions on a facecentered cubic lattice. Here, there are strongly bound Skyrmions that arise when a layer of the face-centered cubic lattice is filled. The Skyrmion with one extra baryon is then described by a core + particle system. To quantize these, Manton suggested that one should allow the extra Skyrmion to only take positions on the next layer of the face-centered cubic lattice [28]. One must still quantize the overall spin and isospin of the system, and our framework shows how to do so. It amounts to replacing the classical configuration space

$$
S O(3)_{J} \times S O(3)_{I} \times \frac{S O(3)_{S} \times S O(3)_{T}}{U(1)}
$$

with

$$
S O(3)_{J} \times S O(3)_{I} \times C_{n},
$$

where $C_{n}$ is a collection of $n$ positions that the additional Skyrmion may take: those on the next layer of the facecentered cubic lattice. The Hamiltonian is then a hopping Hamiltonian, and the relative part of the wave function is a function on a finite set of points. The overall wave functions must still obey the FR constraints discussed in this paper, but now the symmetry transformations are members of the symmetric group of $n$ points, rather than rotations in $S O(3)_{S} \times S O(3)_{T}$. This reduces the complexity of the problem as there is no longer a need to generate a potential on the relative space and the Schrödinger equation is likely exactly solvable. With this simplicity, one may then try to study more complex systems such as those with more than one orbiting nucleon. These include most of the halo nuclei and those with a few more nucleons than a magic nucleus. This quantization may even be relevant for the standard Skyrme model. For example, in the $B=5$ sector, we could restrict the $B=1$ to only take positions at the minima of the interaction potential between the $B=1$ and $B=4$ clusters. In our case, this is at the faces, edges, and corners of the cube (with the $B=1$ orientated in the attractive channel). This will correspond to the tight binding limit rather than the weakly interacting limit we have considered in this paper. It would be interesting to compare the resulting spectra in each limit.

The initial results of this paper are not very promising, but the model can be improved in many ways. To match data, we will need to rely on the existence of the $D_{2}$ symmetric $B=5$ Skyrmion. However, our configuration space does not include it. We were able to find free wave functions that do satisfy the constraint arising from the $D_{2}$-symmetric Skyrmion. However, after inclusion of the potential energy, these were mixed with states that do not. Hence, the overall state only approximately satisfies the constraint. This inconsistency is ultimately due to our exclusion of the $D_{2}$ configuration. We should take account of the mode seen in Fig. 6. However, this evolution is only half of a vibrational mode. The full mode is seen in Fig. 8.

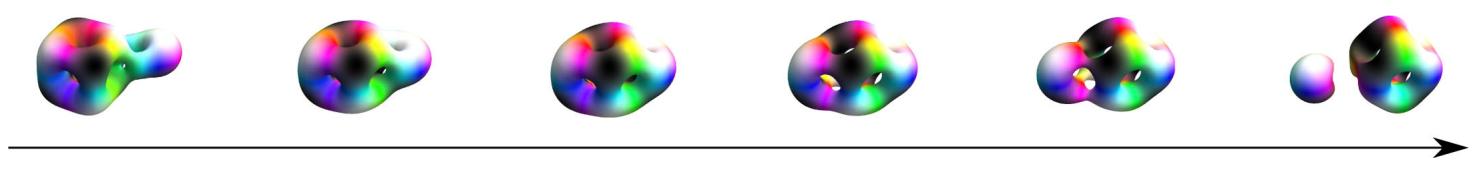

FIG. 8. A vibrational mode of the $4+1$ system. 
A $B=1$ Skyrmion is positioned at an edge of the $B=4$ cube. It merges with the cube to become the $D_{2}$ Skyrmion, but as this process continues, a different $B=1$ Skyrmion is knocked out of the system. The original $B=1$ has become part of a leftover cube, which is rotated and isorotated relative to the original cube.

This novel vibrational mode, which creates a nontrivial relationship between the initial and final configurations of Fig. 8, should be included in the configuration space of the full model. Our calculation knows nothing of the nontrivial relationship, and hence we cannot consistently create nonfree wave functions that are permitted by the $D_{2}$ symmetry. The work of this paper will serve as an essential initial step to understanding the full model. In particular, the wave functions calculated here can be used as part of the eigenfunction basis for the more complicated system. Before progress can be made, a careful study of the $B=$ 5 vibrational space must be completed.

\section{CONCLUSION}

We have attempted to describe the ${ }^{5} \mathrm{He} /{ }^{5} \mathrm{Li}$ isodoublet in the Skyrme model by treating the $B=5$ Skyrmion as a two-cluster system. To do so, we found coordinates in which the overall rotations and isorotations are factored out of the full configuration space. This step is essential for any core + particle system in the Skyrme model. It also drastically reduces the complexity of the problem since the interaction potential only depends on the relative coordinates between the clusters: a five-dimensional space rather than the original 11-dimensional space.

We then constructed wave functions permitted by the cubic symmetry of the $B=4$ core. This construction relied on the representation theory of the system's constituent symmetry groups. The same construction may be applied to any system in which one considers a configuration space of deformed Skyrmions. The representation theory was not enough to construct energy eigenfunctions of definite parity states, and standard techniques were used to determine these.

Until this point, the work was rather model independent, relying only on the cubic symmetry of the $B=4$ core. To calculate the energy spectrum, we had to choose a specific
Skyrme model to fix the moments of inertia and interaction potential. This was done for the standard Skyrme model as well as the loosely bound Skyrme model. The energy spectra of the free and full systems were calculated and compared to experimental data. Both spectra do include low-lying spin- $1 / 2^{-}$and $-3 / 2^{-}$states and a large gap. However, the results contain approximate parity doubling. This is not seen experimentally. It is possible that one can remedy this problem by including the $D_{2}$-symmetric Skyrmion in the quantization scheme. Our work will serve as a foundation for this much more complicated problem and many other two-cluster systems in the Skyrme model.

\section{ACKNOWLEDGMENTS}

We thank Chris King, Nick Manton, and Jonathan Rawlinson for helpful discussions at the early stages of this project. C. H. would also like to thank Derek Harland for discussions on spin-orbit coupling within the Skyrme model. This work is supported by the National Natural Science Foundation of China (Grant No. 11675223).

\section{APPENDIX A: BASIS WAVE FUNCTIONS}

In this Appendix, we tabulate bases of wave functions that transform as the irreps of the symmetry groups $\mathcal{G}_{J I}$ and $\mathcal{G}_{S T}$. Tables III and IV list sets of wave functions in the $J / I$ space, which transform as explained in Sec. III, for a variety of different $J / I$ values. For aesthetic reasons, it is convenient not to normalize the wave functions. However, the wave functions in the text (used to construct the energy eigenstates) are assumed to be normalized. Since the symmetry groups are homomorphic, a table for the $\Phi_{i}$ wave functions on the $S / T$ space would be almost identical. In our conventions, the coefficients of the $S / T$ states are complex conjugates to those of the $J / I$ states.

A permissible wave function takes the form

$$
|\Psi\rangle=\sum_{i} \Phi_{i}\left|\Psi_{i}\right\rangle
$$

where the two sets of wave functions fall into the same irrep. For example, the $\left|\frac{3}{2} \frac{1}{2} \frac{1}{2} \frac{3}{2}, \mathbf{2}^{+}\right\rangle$wave function is

$$
\begin{aligned}
\left|\frac{3}{2} \frac{1}{2} \frac{1}{2} \frac{3}{2} ; \mathbf{2}^{+}\right\rangle= & \left(\left|\frac{3}{2} \frac{3}{2}\right\rangle\left|\frac{1}{2} \frac{1}{2}\right\rangle+\left|\frac{3}{2} \frac{3}{2}\right\rangle\left|\frac{1}{2}-\frac{1}{2}\right\rangle+i\left|\frac{3}{2}-\frac{1}{2}\right\rangle\left|\frac{1}{2} \frac{1}{2}\right\rangle-i\left|\frac{3}{2}-\frac{1}{2}\right\rangle\left|\frac{1}{2}-\frac{1}{2}\right\rangle\right) \\
& \times\left(\left|\frac{1}{2}-\frac{1}{2}\right\rangle\left|\frac{3}{2} \frac{3}{2}\right\rangle+\left|\frac{1}{2}-\frac{1}{2}\right\rangle\left|\frac{3}{2}-\frac{3}{2}\right\rangle\right) \\
& +\left(\left|\frac{3}{2} \frac{1}{2}\right\rangle\left|\frac{1}{2} \frac{1}{2}\right\rangle-i\left|\frac{3}{2} \frac{1}{2}\right\rangle\left|\frac{1}{2}-\frac{1}{2}\right\rangle-i\left|\frac{3}{2}-\frac{3}{2}\right\rangle\left|\frac{1}{2} \frac{1}{2}\right\rangle-i\left|\frac{3}{2}-\frac{3}{2}\right\rangle\left|\frac{1}{2}-\frac{1}{2}\right\rangle\right) \\
& \times\left(-i\left|\frac{1}{2} \frac{1}{2}\right\rangle\left|\frac{3}{2} \frac{3}{2}\right\rangle-i\left|\frac{1}{2} \frac{1}{2}\right\rangle\left|\frac{3}{2}-\frac{3}{2}\right\rangle\right) .
\end{aligned}
$$


TABLE III. Sets of spin states that transform under the irreps of the symmetry group $\mathcal{G}_{J I}$. These are chosen to transform as the realization of the irreps detailed in Sec. IV. The factor of $\mathcal{N}$ represents the fact that the wave functions in the table are not normalized, though the ones used in the text are assumed to be.

\begin{tabular}{|c|c|c|c|}
\hline$\underline{(J, I)}$ & Irrep & $i$ & $\mathcal{N}\left|\Psi_{i}\right\rangle=\sum a_{j_{3} i_{3}}\left|J j_{3}\right\rangle\left|I i_{3}\right\rangle$ \\
\hline \multirow[t]{4}{*}{$\left(\frac{1}{2} \frac{1}{2}\right)$} & 4 & 1 & $\left|\frac{1}{2} \frac{1}{2}\right\rangle\left|\frac{1}{2}-\frac{1}{2}\right\rangle$ \\
\hline & & 2 & $-\left|\frac{1}{2} \frac{1}{2}\right\rangle\left|\frac{1}{2} \frac{1}{2}\right\rangle$ \\
\hline & & 3 & $-i\left|\frac{1}{2}-\frac{1}{2}\right\rangle\left|\frac{1}{2}-\frac{1}{2}\right\rangle$ \\
\hline & & 4 & $i\left|\frac{1}{2}-\frac{1}{2}\right\rangle\left|\frac{1}{2} \frac{1}{2}\right\rangle$ \\
\hline \multirow{8}{*}{$\left(\frac{3}{2} \frac{1}{2}\right)$} & 4 & 1 & $\left|\frac{3}{2} \frac{1}{2}\right\rangle\left|\frac{1}{2} \frac{1}{2}\right\rangle+i\left|\frac{3}{2}-\frac{3}{2}\right\rangle\left|\frac{1}{2} \frac{1}{2}\right\rangle$ \\
\hline & & 2 & $-\left|\frac{3}{2} \frac{1}{2}\right\rangle\left|\frac{1}{2}-\frac{1}{2}\right\rangle+i\left|\frac{3}{2}-\frac{3}{2}\right\rangle\left|\frac{1}{2}-\frac{1}{2}\right\rangle$ \\
\hline & & 3 & $-\left|\frac{3}{2} \frac{3}{2}\right\rangle\left|\frac{1}{2} \frac{1}{2}\right\rangle+i\left|\frac{3}{2}-\frac{1}{2}\right\rangle\left|\frac{1}{2} \frac{1}{2}\right\rangle$ \\
\hline & & 4 & $-\left|\frac{3}{2} \frac{3}{2}\right\rangle\left|\frac{1}{2}-\frac{1}{2}\right\rangle-i\left|\frac{3}{2}-\frac{1}{2}\right\rangle\left|\frac{1}{2}-\frac{1}{2}\right\rangle$ \\
\hline & $\mathbf{2}^{+}$ & 1 & $\left|\frac{3}{2} \frac{3}{2}\right\rangle\left|\frac{1}{2} \frac{1}{2}\right\rangle+\left|\frac{3}{2} \frac{3}{2}\right\rangle\left|\frac{1}{2}-\frac{1}{2}\right\rangle+i\left|\frac{3}{2}-\frac{1}{2}\right\rangle\left|\frac{1}{2} \frac{1}{2}\right\rangle-i\left|\frac{3}{2}-\frac{1}{2}\right\rangle\left|\frac{1}{2}-\frac{1}{2}\right\rangle$ \\
\hline & & 2 & $\left|\frac{3}{2} \frac{1}{2}\right\rangle\left|\frac{1}{2} \frac{1}{2}\right\rangle-i\left|\frac{3}{2} \frac{1}{2}\right\rangle\left|\frac{1}{2}-\frac{1}{2}\right\rangle-i\left|\frac{3}{2}-\frac{3}{2}\right\rangle\left|\frac{1}{2} \frac{1}{2}\right\rangle-i\left|\frac{3}{2}-\frac{3}{2}\right\rangle\left|\frac{1}{2}-\frac{1}{2}\right\rangle$ \\
\hline & $2^{-}$ & 1 & $\left|\frac{3}{2} \frac{3}{2}\right\rangle\left|\frac{1}{2} \frac{1}{2}\right\rangle-\left|\frac{3}{2} \frac{3}{2}\right\rangle\left|\frac{1}{2}-\frac{1}{2}\right\rangle+i\left|\frac{3}{2}-\frac{1}{2}\right\rangle\left|\frac{1}{2} \frac{1}{2}\right\rangle+i\left|\frac{3}{2}-\frac{1}{2}\right\rangle\left|\frac{1}{2}-\frac{1}{2}\right\rangle$ \\
\hline & & 2 & $\left|\frac{3}{2} \frac{1}{2}\right\rangle\left|\frac{1}{2} \frac{1}{2}\right\rangle+i\left|\frac{3}{2} \frac{1}{2}\right\rangle\left|\frac{1}{2}-\frac{1}{2}\right\rangle-i\left|\frac{3}{2}-\frac{3}{2}\right\rangle\left|\frac{1}{2} \frac{1}{2}\right\rangle+i\left|\frac{3}{2}-\frac{3}{2}\right\rangle\left|\frac{1}{2}-\frac{1}{2}\right\rangle$ \\
\hline \multirow[t]{12}{*}{$\left(\frac{5}{2} \frac{1}{2}\right)$} & $4_{1}$ & 1 & $\left|\frac{5}{2} \frac{5}{2}\right\rangle\left|\frac{1}{2} \frac{1}{2}\right\rangle$ \\
\hline & & 2 & $-\left|\frac{5}{2} \frac{1}{2}\right\rangle\left|\frac{1}{2}-\frac{1}{2}\right\rangle$ \\
\hline & & 3 & $-i\left|\frac{5}{2}-\frac{1}{2}\right\rangle\left|\frac{1}{2} \frac{1}{2}\right\rangle$ \\
\hline & & 4 & $i\left|\frac{5}{2}-\frac{5}{2}\right\rangle\left|\frac{1}{2}-\frac{1}{2}\right\rangle$ \\
\hline & $\mathbf{4}_{2}$ & 1 & $\left|\frac{5}{2} \frac{3}{2}\right\rangle\left|\frac{1}{2}-\frac{1}{2}\right\rangle$ \\
\hline & & 2 & $-\left|\frac{5}{2} \frac{3}{2}\right\rangle\left|\frac{1}{2} \frac{1}{2}\right\rangle$ \\
\hline & & 3 & $-i\left|\frac{5}{2}-\frac{3}{2}\right\rangle\left|\frac{1}{2}-\frac{1}{2}\right\rangle$ \\
\hline & & 4 & $i\left|\frac{5}{2}-\frac{3}{2}\right\rangle\left|\frac{1}{2} \frac{1}{2}\right\rangle$ \\
\hline & $2^{+}$ & 1 & $\left|\frac{5}{2}-\frac{1}{2}\right\rangle\left|\frac{1}{2}-\frac{1}{2}\right\rangle-\left|\frac{5}{2}-\frac{5}{2}\right\rangle\left|\frac{1}{2} \frac{1}{2}\right\rangle$ \\
\hline & & 2 & $i\left|\frac{5}{2} \frac{5}{2}\right\rangle\left|\frac{1}{2}-\frac{1}{2}\right\rangle-i\left|\frac{5}{2} \frac{1}{2}\right\rangle\left|\frac{1}{2} \frac{1}{2}\right\rangle$ \\
\hline & $2^{-}$ & 1 & $\left|\frac{5}{2}-\frac{1}{2}\right\rangle\left|\frac{1}{2}-\frac{1}{2}\right\rangle+\left|\frac{5}{2}-\frac{5}{2}\right\rangle\left|\frac{1}{2} \frac{1}{2}\right\rangle$ \\
\hline & & 2 & $i\left|\frac{5}{2} \frac{5}{2}\right\rangle\left|\frac{1}{2}-\frac{1}{2}\right\rangle+i\left|\frac{5}{2} \frac{1}{2}\right\rangle\left|\frac{1}{2} \frac{1}{2}\right\rangle$ \\
\hline \multirow[t]{8}{*}{$\left(\frac{1}{2} \frac{3}{2}\right)$} & 4 & 1 & $\left|\frac{1}{2} \frac{1}{2}\right\rangle\left|\frac{3}{2}-\frac{1}{2}\right\rangle$ \\
\hline & & 2 & $\left|\frac{1}{2} \frac{1}{2}\right\rangle\left|\frac{3}{2} \frac{1}{2}\right\rangle$ \\
\hline & & 3 & $-i\left|\frac{1}{2}-\frac{1}{2}\right\rangle\left|\frac{3}{2}-\frac{1}{2}\right\rangle$ \\
\hline & & 4 & $-i\left|\frac{1}{2}-\frac{1}{2}\right\rangle\left|\frac{3}{2} \frac{1}{2}\right\rangle$ \\
\hline & $2^{+}$ & 1 & $\left|\frac{1}{2}-\frac{1}{2}\right\rangle\left|\frac{3}{2} \frac{3}{2}\right\rangle+\left|\frac{1}{2}-\frac{1}{2}\right\rangle\left|\frac{3}{2}-\frac{3}{2}\right\rangle$ \\
\hline & & 2 & $i\left|\frac{1}{2} \frac{1}{2}\right\rangle\left|\frac{3}{2} \frac{3}{2}\right\rangle+i\left|\frac{1}{2} \frac{1}{2}\right\rangle\left|\frac{3}{2}-\frac{3}{2}\right\rangle$ \\
\hline & $2^{-}$ & 1 & $\left|\frac{1}{2}-\frac{1}{2}\right\rangle\left|\frac{3}{2} \frac{3}{2}\right\rangle-\left|\frac{1}{2}-\frac{1}{2}\right\rangle\left|\frac{3}{2}-\frac{3}{2}\right\rangle$ \\
\hline & & 2 & $i\left|\frac{1}{2} \frac{1}{2}\right\rangle\left|\frac{3}{2} \frac{3}{2}\right\rangle-i\left|\frac{1}{2} \frac{1}{2}\right\rangle\left|\frac{3}{2}-\frac{3}{2}\right\rangle$ \\
\hline
\end{tabular}

We have suppressed the space-fixed projection for aesthetic reasons. We can then use these wave functions to generate the energy eigenfunctions.

\section{APPENDIX B: ENERGY EIGENSTATES}

Low-energy eigenstates were mentioned in the main text. Here, we tabulate several higher-energy states, including all of those used in Fig. 7, in Tables IV and V. We list them in order of increasing energy. In this table, we assume that the constituent states have been normalized.
In the search for low-energy states, we considered a wide variety of different $(J, I, S, T)$ combinations. For $(J, I)=$ $(1 / 2,1 / 2)$ and $(J, I)=(3 / 2,1 / 2)$, we considered

$$
\begin{gathered}
(S, T)=\left(\left(\frac{1}{2}, \frac{1}{2}\right),\left(\frac{3}{2}, \frac{1}{2}\right),\left(\frac{5}{2}, \frac{1}{2}\right),\left(\frac{7}{2}, \frac{1}{2}\right),\right. \\
\left.\left(\frac{1}{2}, \frac{3}{2}\right),\left(\frac{3}{2}, \frac{3}{2}\right),\left(\frac{1}{2}, \frac{5}{2}\right)\right) .
\end{gathered}
$$

For the higher spins and isospins, 
TABLE IV. More spin-state bases, for $(J, I)=(3 / 2,3 / 2)$ and $(J, I)=(7 / 2,1 / 2)$, which transform under the irreps of $\mathcal{G}_{J I}$.

\begin{tabular}{|c|c|c|}
\hline Irrep & $i$ & $\mathcal{N}\left|\Psi_{i}\right\rangle=\sum a_{j_{3} i_{3}}\left|J j_{3}\right\rangle\left|I i_{3}\right\rangle$ \\
\hline \multirow[t]{4}{*}{$4_{1}$} & 1 & $\left|\frac{3}{2} \frac{1}{2}\right\rangle\left|\frac{3}{2} \frac{3}{2}\right\rangle-i\left|\frac{3}{2}-\frac{3}{2}\right\rangle\left|\frac{3}{2} \frac{3}{2}\right\rangle$ \\
\hline & 2 & $\left|\frac{3}{2} \frac{1}{2}\right\rangle\left|\frac{3}{2}-\frac{3}{2}\right\rangle+i\left|\frac{3}{2}-\frac{3}{2}\right\rangle\left|\frac{3}{2}-\frac{3}{2}\right\rangle$ \\
\hline & 3 & $\left|\frac{3}{2} \frac{3}{2}\right\rangle\left|\frac{3}{2} \frac{3}{2}\right\rangle+i\left|\frac{3}{2}-\frac{1}{2}\right\rangle\left|\frac{3}{2} \frac{3}{2}\right\rangle$ \\
\hline & 4 & $-\left|\frac{3}{2} \frac{3}{2}\right\rangle\left|\frac{3}{2}-\frac{3}{2}\right\rangle+i\left|\frac{3}{2}-\frac{1}{2}\right\rangle\left|\frac{3}{2}-\frac{3}{2}\right\rangle$ \\
\hline \multirow[t]{4}{*}{$\mathbf{4}_{2}$} & 1 & $\left|\frac{3}{2} \frac{1}{2}\right\rangle\left|\frac{3}{2} \frac{1}{2}\right\rangle+i\left|\frac{3}{2}-\frac{3}{2}\right\rangle\left|\frac{3}{2} \frac{1}{2}\right\rangle$ \\
\hline & 2 & $\left|\frac{3}{2} \frac{1}{2}\right\rangle\left|\frac{3}{2}-\frac{1}{2}\right\rangle-i\left|\frac{3}{2}-\frac{3}{2}\right\rangle\left|\frac{3}{2}-\frac{1}{2}\right\rangle$ \\
\hline & 3 & $-\left|\frac{3}{2} \frac{3}{2}\right\rangle\left|\frac{3}{2} \frac{1}{2}\right\rangle+i\left|\frac{3}{2} \frac{1}{2}\right\rangle\left|\frac{3}{2} \frac{1}{2}\right\rangle$ \\
\hline & 4 & $\left|\frac{3}{2} \frac{3}{2}\right\rangle\left|\frac{3}{2}-\frac{1}{2}\right\rangle+i\left|\frac{3}{2}-\frac{1}{2}\right\rangle\left|\frac{3}{2}-\frac{1}{2}\right\rangle$ \\
\hline \multirow[t]{4}{*}{$4_{3}$} & 1 & $\left|\frac{3}{2} \frac{1}{2}\right\rangle\left|\frac{3}{2}-\frac{3}{2}\right\rangle-i\left|\frac{3}{2}-\frac{3}{2}\right\rangle\left|\frac{3}{2}-\frac{3}{2}\right\rangle$ \\
\hline & 2 & $\left|\frac{3}{2} \frac{1}{2}\right\rangle\left|\frac{3}{2} \frac{3}{2}\right\rangle+i\left|\frac{3}{2}-\frac{3}{2}\right\rangle\left|\frac{3}{2} \frac{3}{2}\right\rangle$ \\
\hline & 3 & $\left|\frac{3}{2} \frac{3}{2}\right\rangle\left|\frac{3}{2}-\frac{3}{2}\right\rangle+i\left|\frac{3}{2}-\frac{1}{2}\right\rangle\left|\frac{3}{2}-\frac{3}{2}\right\rangle$ \\
\hline & 4 & $-\left|\frac{3}{2} \frac{3}{2}\right\rangle\left|\frac{3}{2} \frac{3}{2}\right\rangle+i\left|\frac{3}{2}-\frac{1}{2}\right\rangle\left|\frac{3}{2} \frac{3}{2}\right\rangle$ \\
\hline \multirow[t]{2}{*}{$2^{+}$} & 1 & $\left|\frac{3}{2} \frac{3}{2}\right\rangle\left|\frac{3}{2} \frac{1}{2}\right\rangle+\left|\frac{3}{2} \frac{3}{2}\right\rangle\left|\frac{3}{2}-\frac{1}{2}\right\rangle+i\left|\frac{3}{2}-\frac{1}{2}\right\rangle\left|\frac{3}{2} \frac{1}{2}\right\rangle-i\left|\frac{3}{2}-\frac{1}{2}\right\rangle\left|\frac{3}{2}-\frac{1}{2}\right\rangle$ \\
\hline & 2 & $\left|\frac{3}{2} \frac{1}{2}\right\rangle\left|\frac{3}{2} \frac{1}{2}\right\rangle-\left|\frac{3}{2} \frac{1}{2}\right\rangle\left|\frac{3}{2}-\frac{1}{2}\right\rangle-i\left|\frac{3}{2}-\frac{3}{2}\right\rangle\left|\frac{3}{2} \frac{1}{2}\right\rangle-i\left|\frac{3}{2}-\frac{3}{2}\right\rangle\left|\frac{3}{2}-\frac{1}{2}\right\rangle$ \\
\hline \multirow[t]{2}{*}{$2^{-}$} & 1 & $\left|\frac{3}{2} \frac{3}{2}\right\rangle\left|\frac{3}{2} \frac{1}{2}\right\rangle-\left|\frac{3}{2} \frac{3}{2}\right\rangle\left|\frac{3}{2}-\frac{1}{2}\right\rangle+i\left|\frac{3}{2}-\frac{1}{2}\right\rangle\left|\frac{3}{2} \frac{1}{2}\right\rangle+i\left|\frac{3}{2}-\frac{1}{2}\right\rangle\left|\frac{3}{2}-\frac{1}{2}\right\rangle$ \\
\hline & 2 & $\left|\frac{3}{2} \frac{1}{2}\right\rangle\left|\frac{3}{2} \frac{1}{2}\right\rangle+\left|\frac{3}{2} \frac{1}{2}\right\rangle\left|\frac{3}{2}-\frac{1}{2}\right\rangle-i\left|\frac{3}{2}-\frac{3}{2}\right\rangle\left|\frac{3}{2} \frac{1}{2}\right\rangle+i\left|\frac{3}{2}-\frac{3}{2}\right\rangle\left|\frac{3}{2}-\frac{1}{2}\right\rangle$ \\
\hline \multirow[t]{4}{*}{$4_{1}$} & 1 & $\sqrt{3}\left|\frac{7}{2} \frac{5}{2}\right\rangle\left|\frac{1}{2} \frac{1}{2}\right\rangle+i \sqrt{5}\left|\frac{7}{2} \frac{1}{2}\right\rangle\left|\frac{1}{2} \frac{1}{2}\right\rangle+3\left|\frac{7}{2}-\frac{3}{2}\right\rangle\left|\frac{1}{2} \frac{1}{2}\right\rangle-i \sqrt{7}\left|\frac{7}{2}-\frac{7}{2}\right\rangle\left|\frac{1}{2} \frac{1}{2}\right\rangle$ \\
\hline & 2 & $\sqrt{3}\left|\frac{7}{2} \frac{5}{2}\right\rangle\left|\frac{1}{2}-\frac{1}{2}\right\rangle-i \sqrt{5}\left|\frac{7}{2} \frac{1}{2}\right\rangle\left|\frac{1}{2}-\frac{1}{2}\right\rangle+3\left|\frac{7}{2}-\frac{3}{2}\right\rangle\left|\frac{1}{2}-\frac{1}{2}\right\rangle+i \sqrt{7}\left|\frac{7}{2}-\frac{7}{2}\right\rangle\left|\frac{1}{2}-\frac{1}{2}\right\rangle$ \\
\hline & 3 & $\sqrt{7}\left|\frac{7}{2} \frac{7}{2}\right\rangle\left|\frac{1}{2} \frac{1}{2}\right\rangle+3 i\left|\frac{7}{2} \frac{3}{2}\right\rangle\left|\frac{1}{2} \frac{1}{2}\right\rangle-\sqrt{5}\left|\frac{7}{2}-\frac{1}{2}\right\rangle\left|\frac{1}{2} \frac{1}{2}\right\rangle+i \sqrt{3}\left|\frac{7}{2}-\frac{5}{2}\right\rangle\left|\frac{1}{2} \frac{1}{2}\right\rangle$ \\
\hline & 4 & $-\sqrt{7}\left|\frac{7}{2} \frac{7}{2}\right\rangle\left|\frac{1}{2}-\frac{1}{2}\right\rangle+3 i\left|\frac{7}{2} \frac{3}{2}\right\rangle\left|\frac{1}{2}-\frac{1}{2}\right\rangle+\sqrt{5}\left|\frac{7}{2}-\frac{1}{2}\right\rangle\left|\frac{1}{2}-\frac{1}{2}\right\rangle+i \sqrt{3}\left|\frac{7}{2}-\frac{5}{2}\right\rangle\left|\frac{1}{2}-\frac{1}{2}\right\rangle$ \\
\hline \multirow[t]{4}{*}{$\mathbf{4}_{2}$} & 1 & $\sqrt{3}\left|\frac{7}{2} \frac{5}{2}\right\rangle\left|\frac{1}{2}-\frac{1}{2}\right\rangle-\left|\frac{7}{2}-\frac{3}{2}\right\rangle\left|\frac{1}{2}-\frac{1}{2}\right\rangle$ \\
\hline & 2 & $\sqrt{3}\left|\frac{7}{2} \frac{5}{2}\right\rangle\left|\frac{1}{2} \frac{1}{2}\right\rangle-\left|\frac{7}{2}-\frac{3}{2}\right\rangle\left|\frac{1}{2} \frac{1}{2}\right\rangle$ \\
\hline & 3 & $-i\left|\frac{7}{2} \frac{3}{2}\right\rangle\left|\frac{1}{2}-\frac{1}{2}\right\rangle+i \sqrt{3}\left|\frac{7}{2}-\frac{5}{2}\right\rangle\left|\frac{1}{2}-\frac{1}{2}\right\rangle$ \\
\hline & 4 & $-i\left|\frac{7}{2} \frac{3}{2}\right\rangle\left|\frac{1}{2} \frac{1}{2}\right\rangle+i \sqrt{3}\left|\frac{7}{2}-\frac{5}{2}\right\rangle\left|\frac{1}{2} \frac{1}{2}\right\rangle$ \\
\hline \multirow[t]{4}{*}{$\mathbf{4}_{3}$} & 1 & $\sqrt{7}\left|\frac{7}{2} \frac{1}{2}\right\rangle\left|\frac{1}{2}-\frac{1}{2}\right\rangle-\sqrt{5}\left|\frac{7}{2}-\frac{7}{2}\right\rangle\left|\frac{1}{2}-\frac{1}{2}\right\rangle$ \\
\hline & 2 & $-\sqrt{7}\left|\frac{7}{2} \frac{1}{2}\right\rangle\left|\frac{1}{2} \frac{1}{2}\right\rangle-\sqrt{5}\left|\frac{7}{2}-\frac{7}{2}\right\rangle\left|\frac{1}{2} \frac{1}{2}\right\rangle$ \\
\hline & 3 & $i \sqrt{5}\left|\frac{7}{2} \frac{7}{2}\right\rangle\left|\frac{1}{2}-\frac{1}{2}\right\rangle+i \sqrt{7}\left|\frac{7}{2}-\frac{1}{2}\right\rangle\left|\frac{1}{2}-\frac{1}{2}\right\rangle$ \\
\hline & 4 & $-i \sqrt{5}\left|\frac{7}{2} \frac{7}{2}\right\rangle\left|\frac{1}{2} \frac{1}{2}\right\rangle-i \sqrt{7}\left|\frac{7}{2}-\frac{1}{2}\right\rangle\left|\frac{1}{2} \frac{1}{2}\right\rangle$ \\
\hline \multirow[t]{2}{*}{$2^{+}$} & 1 & $\begin{array}{c}\sqrt{7}\left|\frac{7}{2} \frac{7}{2}\right\rangle\left|\frac{1}{2} \frac{1}{2}\right\rangle-\sqrt{7}\left|\frac{7}{2} \frac{7}{2}\right\rangle\left|\frac{1}{2}-\frac{1}{2}\right\rangle-3 i\left|\frac{7}{2} \frac{3}{2}\right\rangle\left|\frac{1}{2} \frac{1}{2}\right\rangle-3 i\left|\frac{7}{2} \frac{3}{2}\right\rangle\left|\frac{1}{2}-\frac{1}{2}\right\rangle- \\
\sqrt{5}\left|\frac{7}{2}-\frac{1}{2}\right\rangle\left|\frac{1}{2} \frac{1}{2}\right\rangle+\sqrt{5}\left|\frac{7}{2}-\frac{1}{2}\right\rangle\left|\frac{1}{2}-\frac{1}{2}\right\rangle-i \sqrt{3}\left|\frac{7}{2}-\frac{5}{2}\right\rangle\left|\frac{1}{2} \frac{1}{2}\right\rangle-i \sqrt{3}\left|\frac{7}{2}-\frac{5}{2}\right\rangle\left|\frac{1}{2}-\frac{1}{2}\right\rangle\end{array}$ \\
\hline & 2 & $\begin{array}{c}-\sqrt{3}\left|\frac{7}{2} \frac{5}{2}\right\rangle\left|\frac{1}{2} \frac{1}{2}\right\rangle-\sqrt{3}\left|\frac{7}{2} \frac{5}{2}\right\rangle\left|\frac{1}{2}-\frac{1}{2}\right\rangle+i \sqrt{5}\left|\frac{7}{2} \frac{1}{2}\right\rangle\left|\frac{1}{2} \frac{1}{2}\right\rangle-i \sqrt{5}\left|\frac{7}{2} \frac{1}{2}\right\rangle\left|\frac{1}{2}-\frac{1}{2}\right\rangle- \\
3\left|\frac{7}{2}-\frac{3}{2}\right\rangle\left|\frac{1}{2} \frac{1}{2}\right\rangle-3\left|\frac{7}{2}-\frac{3}{2}\right\rangle\left|\frac{1}{2}-\frac{1}{2}\right\rangle-i \sqrt{7}\left|\frac{7}{2}-\frac{7}{2}\right\rangle\left|\frac{1}{2} \frac{1}{2}\right\rangle+i \sqrt{7}\left|\frac{7}{2}-\frac{7}{2}\right\rangle\left|\frac{1}{2}-\frac{1}{2}\right\rangle\end{array}$ \\
\hline \multirow[t]{2}{*}{$2^{-}$} & 1 & $\begin{array}{c}\sqrt{7}\left|\frac{7}{2} \frac{7}{2}\right\rangle\left|\frac{1}{2} \frac{1}{2}\right\rangle+\sqrt{7}\left|\frac{7}{2} \frac{7}{2}\right\rangle\left|\frac{1}{2}-\frac{1}{2}\right\rangle-3 i\left|\frac{7}{2} \frac{3}{2}\right\rangle\left|\frac{1}{2} \frac{1}{2}\right\rangle+3 i\left|\frac{7}{2} \frac{3}{2}\right\rangle\left|\frac{1}{2}-\frac{1}{2}\right\rangle- \\
\sqrt{5}\left|\frac{7}{2}-\frac{1}{2}\right\rangle\left|\frac{1}{2} \frac{1}{2}\right\rangle-\sqrt{5}\left|\frac{7}{2}-\frac{1}{2}\right\rangle\left|\frac{1}{2}-\frac{1}{2}\right\rangle-i \sqrt{3}\left|\frac{7}{2}-\frac{5}{2}\right\rangle\left|\frac{1}{2} \frac{1}{2}\right\rangle+i \sqrt{3}\left|\frac{7}{2}-\frac{5}{2}\right\rangle\left|\frac{1}{2}-\frac{1}{2}\right\rangle\end{array}$ \\
\hline & 2 & $\begin{array}{c}-\sqrt{3}\left|\frac{7}{2} \frac{5}{2}\right\rangle\left|\frac{1}{2} \frac{1}{2}\right\rangle+\sqrt{3}\left|\frac{7}{2} \frac{5}{2}\right\rangle\left|\frac{1}{2}-\frac{1}{2}\right\rangle+i \sqrt{5}\left|\frac{7}{2} \frac{1}{2}\right\rangle\left|\frac{1}{2} \frac{1}{2}\right\rangle+i \sqrt{5}\left|\frac{7}{2} \frac{1}{2}\right\rangle\left|\frac{1}{2}-\frac{1}{2}\right\rangle- \\
3\left|\frac{7}{2}-\frac{3}{2}\right\rangle\left|\frac{1}{2} \frac{1}{2}\right\rangle+3\left|\frac{7}{2}-\frac{3}{2}\right\rangle\left|\frac{1}{2}-\frac{1}{2}\right\rangle-i \sqrt{7}\left|\frac{7}{2}-\frac{7}{2}\right\rangle\left|\frac{1}{2} \frac{1}{2}\right\rangle-i \sqrt{7}\left|\frac{7}{2}-\frac{7}{2}\right\rangle\left|\frac{1}{2}-\frac{1}{2}\right\rangle\end{array}$ \\
\hline
\end{tabular}


TABLE V. The 19 lowest-energy eigenfunctions and their corresponding energies. We use the notation $\left|J I S T ; \boldsymbol{X}_{\boldsymbol{i}}\right\rangle$ as described in Sec. IV. We omit $t_{3}^{\prime}$ as this has no effect on the energy.

\begin{tabular}{|c|c|}
\hline Energy eigenstate & Energy \\
\hline$\left|\frac{1}{2} \frac{1}{2} \frac{1}{2} \frac{1}{2} ; 4\right\rangle$ & $\frac{3}{8 v_{1}}+\frac{3}{4 \mu}$ \\
\hline$\sqrt{2}\left|\frac{3}{2} \frac{1}{2} \frac{3}{2} \frac{1}{2} ; \mathbf{4}\right\rangle+\left|\frac{3}{2} \frac{1}{2} \frac{3}{2} \frac{1}{2} ; \mathbf{2}^{+}\right\rangle+\left|\frac{3}{2} \frac{1}{2} \frac{3}{2} \frac{1}{2} ; \mathbf{2}^{-}\right\rangle$ & $\frac{3}{8 v_{1}}+\frac{9}{4 \mu}$ \\
\hline $\begin{array}{l}\left|\frac{3}{2} \frac{1}{2} \frac{1}{2} \frac{1}{2} ; \mathbf{4}\right\rangle \\
\left|\frac{5}{2} \frac{1}{2} \frac{1}{2} \frac{1}{2} ; \mathbf{4}_{1}\right\rangle \\
\left|\frac{3}{2} \frac{3}{2} \frac{1}{2} \frac{1}{2} ; \mathbf{4}_{2}\right\rangle-\sqrt{3}\left|\frac{3}{2} \frac{3}{2} \frac{1}{2} \frac{1}{2} ; \mathbf{4}_{3}\right\rangle\end{array}$ & $\begin{array}{l}\frac{3}{8 v_{1}}+\frac{1}{2 u_{11}}+\frac{1}{2 u_{33}}+\frac{3}{v_{4}}+\frac{3}{4 \mu} \\
\frac{3}{8 v_{1}}+\frac{1}{2 u_{11}}+\frac{1}{2 u_{33}}+\frac{3}{v_{4}}+\frac{3}{4 \mu} \\
\frac{3}{8 v_{1}}+\frac{1}{2 u_{11}}+\frac{1}{2 u_{33}}+\frac{3}{v_{4}}+\frac{3}{4 \mu}\end{array}$ \\
\hline $\begin{array}{l}\left|\frac{1}{2} \frac{1}{2} \frac{3}{2} \frac{1}{2} ; \mathbf{4}\right\rangle \\
\left|\frac{3}{2} \frac{1}{2} \frac{3}{2} \frac{1}{2} ; \mathbf{2}^{+}\right\rangle-\left|\frac{3}{2} \frac{1}{2} \frac{3}{2} \frac{1}{2} ; \mathbf{2}^{-}\right\rangle \\
i \sqrt{5}\left|\frac{5}{2} \frac{1}{2} \frac{3}{2} \frac{1}{2} ; \mathbf{4}_{\mathbf{2}}\right\rangle-\left|\frac{5}{2} \frac{1}{2} \frac{3}{2} \frac{1}{2} ; \mathbf{2}^{+}\right\rangle+\left|\frac{5}{2} \frac{1}{2} \frac{3}{2} \frac{1}{2} ; \mathbf{2}^{-}\right\rangle \\
\left(\sqrt{5}\left|\frac{7}{2} \frac{1}{2} \frac{3}{2} \frac{1}{2} ; \mathbf{4}_{\mathbf{2}}\right\rangle+i \sqrt{14}\left|\frac{7}{2} \frac{1}{2} \frac{3}{2} \frac{1}{2} ; \mathbf{4}_{\mathbf{3}}\right\rangle-\right.\end{array}$ & $\begin{array}{l}\frac{3}{8 v_{1}}+\frac{1}{2 u_{11}}+\frac{1}{2 u_{33}}+\frac{3}{v_{4}}+\frac{9}{4 \mu} \\
\frac{3}{8 v_{1}}+\frac{1}{2 u_{11}}+\frac{1}{2 u_{33}}+\frac{3}{v_{4}}+\frac{9}{4 \mu} \\
\frac{3}{8 v_{1}}+\frac{1}{2 u_{11}}+\frac{1}{2 u_{33}}+\frac{3}{v_{4}}+\frac{9}{4 \mu}\end{array}$ \\
\hline $\begin{array}{l}\left.\sqrt{10}\left|\frac{7}{2} \frac{1}{2} \frac{3}{2} \frac{1}{2} ; \mathbf{2}^{+}\right\rangle+\sqrt{10}\left|\frac{7}{2} \frac{1}{2} \frac{3}{2} \frac{1}{2} ; \mathbf{2}^{-}\right\rangle\right) \\
\sqrt{2}\left|\frac{1}{2} \frac{3}{2} \frac{3}{2} \frac{1}{2} ; \mathbf{4}\right\rangle+\sqrt{3}\left|\frac{1}{2} \frac{3}{2} \frac{3}{2} \frac{1}{2} ; \mathbf{2}^{+}\right\rangle+\sqrt{3}\left|\frac{1}{2} \frac{3}{2} \frac{3}{2} \frac{1}{2} ; \mathbf{2}^{-}\right\rangle\end{array}$ & $\begin{array}{l}\frac{3}{8 v_{1}}+\frac{1}{2 u_{11}}+\frac{1}{2 u_{33}}+\frac{3}{v_{4}}+\frac{9}{4 \mu} \\
\frac{3}{8 v_{1}}+\frac{1}{2 u_{11}}+\frac{1}{2 u_{33}}+\frac{3}{v_{4}}+\frac{9}{4 \mu}\end{array}$ \\
\hline $\begin{array}{l}\left|\frac{1}{2} \frac{1}{2} \frac{5}{2} \frac{1}{2} ; \mathbf{4}_{\mathbf{2}}\right\rangle \\
i \sqrt{5}\left|\frac{3}{2} \frac{1}{2} \frac{5}{2} \frac{1}{2} ; \mathbf{4}_{\mathbf{2}}\right\rangle-\left|\frac{3}{2} \frac{1}{2} \frac{5}{2} \frac{1}{2} ; \mathbf{2}^{+}\right\rangle+\left|\frac{3}{2} \frac{1}{2} \frac{5}{2} \frac{1}{2} ; \mathbf{2}^{-}\right\rangle \\
\left|\frac{7}{2} \frac{1}{2} \frac{1}{2} \frac{1}{2} ; \mathbf{4}_{\mathbf{3}}\right\rangle \\
\left|\frac{3}{2} \frac{3}{2} \frac{3}{2} \frac{1}{2} ; \mathbf{4}_{\mathbf{1}}\right\rangle\end{array}$ & $\begin{array}{l}\frac{3}{8 v_{1}}+\frac{1}{2 u_{11}}+\frac{1}{2 u_{33}}+\frac{3}{v_{4}}+\frac{19}{4 \mu} \\
\frac{3}{8 v_{1}}+\frac{1}{2 u_{11}}+\frac{1}{2 u_{33}}+\frac{3}{v_{4}}+\frac{19}{4 \mu} \\
\frac{3}{8 v_{1}}+\frac{10}{v_{4}}+\frac{3}{4 \mu} \\
\frac{3}{4 v_{1}}+\frac{1}{u_{11}}+\frac{1}{2 u_{33}}+\frac{3}{v_{4}}+\frac{9}{4 \mu}\end{array}$ \\
\hline $\begin{array}{l}\left|\frac{5}{2} \frac{1}{2} \frac{1}{2} \frac{1}{2} ; \mathbf{4}_{1}\right\rangle \\
\left|\frac{7}{2} \frac{1}{2} \frac{1}{2} \frac{1}{2} ; \mathbf{4}_{2}\right\rangle\end{array}$ & $\begin{array}{l}\frac{3}{4 v_{1}}+\frac{1}{u_{11}}+\frac{6}{v_{4}}+\frac{3}{4 \mu} \\
\frac{3}{4 v_{1}}+\frac{1}{u_{11}}+\frac{6}{v_{4}}+\frac{3}{4 \mu}\end{array}$ \\
\hline $\begin{array}{l}-i \sqrt{2}\left|\frac{5}{2} \frac{1}{2} \frac{3}{2} \frac{1}{2} ; \mathbf{4}_{\mathbf{1}}\right\rangle+\left|\frac{5}{2} \frac{1}{2} \frac{3}{2} \frac{1}{2} ; \mathbf{2}^{+}\right\rangle+\left|\frac{5}{2} \frac{1}{2} \frac{3}{2} \frac{1}{2} ; \mathbf{2}^{-}\right\rangle \\
\sqrt{2}\left|\frac{7}{2} \frac{1}{2} \frac{3}{2} \frac{1}{2} ; \mathbf{4}_{\mathbf{1}}\right\rangle+\left|\frac{7}{2} \frac{1}{2} \frac{3}{2} \frac{1}{2} ; \mathbf{2}^{+}\right\rangle+\left|\frac{7}{2} \frac{1}{2} \frac{3}{2} \frac{1}{2} ; \mathbf{2}^{-}\right\rangle \\
\end{array}$ & $\begin{array}{l}\frac{3}{8 v_{1}}+\frac{10}{v_{4}}+\frac{9}{4 \mu} \\
\frac{3}{8 v_{1}}+\frac{10}{v_{4}}+\frac{9}{4 \mu}\end{array}$ \\
\hline
\end{tabular}

$$
(J, I)=\left(\left(\frac{5}{2}, \frac{1}{2}\right),\left(\frac{7}{2}, \frac{1}{2}\right),\left(\frac{1}{2}, \frac{3}{2}\right),\left(\frac{3}{2}, \frac{3}{2}\right)\right),
$$

we only considered $(S, T)=(1 / 2,1 / 2)$ and $(S, T)=(3 / 2,1 / 2)$. In total, we calculated 144 energy eigenstates of the free system.

[1] T. H. R. Skyrme, A nonlinear field theory, Proc. R. Soc. A 260, 127 (1961).

[2] R. Battye, N. S. Manton, and P. Sutcliffe, Skyrmions and the alpha-particle model of nuclei, Proc. R. Soc. A 463, 261 (2007).

[3] O. V. Manko, N. S. Manton, and S. W. Wood, Light nuclei as quantized skyrmions, Phys. Rev. C 76, 055203 (2007).

[4] C. Adam, J. Sanchez-Guillen, and A. Wereszczynski, A Skyrme-type proposal for baryonic matter, Phys. Lett. B 691, 105 (2010).

[5] P. H. C. Lau and N. S. Manton, States of Carbon-12 in the Skyrme Model, Phys. Rev. Lett. 113, 232503 (2014).
[6] C. J. Halcrow, C. King, and N. S. Manton, A dynamical $\alpha$-cluster model of ${ }^{16} \mathrm{O}$, Phys. Rev. C 95, 031303 (2017).

[7] C. J. Halcrow, Vibrational quantisation of the $B=7$ Skyrmion, Nucl. Phys. B904, 106 (2016).

[8] S. B. Gudnason, Loosening up the Skyrme model, Phys. Rev. D 93, 065048 (2016).

[9] S. B. Gudnason and M. Nitta, Modifying the pion mass in the loosely bound Skyrme model, Phys. Rev. D 94, 065018 (2016).

[10] S. B. Gudnason, B. Zhang, and N. Ma, Generalized Skyrme model with the loosely bound potential, Phys. Rev. D 94, 125004 (2016). 
[11] M. Gillard, D. Harland, and M. Speight, Skyrmions with low binding energies, Nucl. Phys. B895, 272 (2015).

[12] M. Gillard, D. Harland, E. Kirk, B. Maybee, and M. Speight, A point particle model of lightly bound skyrmions, Nucl. Phys. B917, 286 (2017).

[13] C. J. Halcrow and N. S. Manton, A Skyrme model approach to the spin-orbit force, J. High Energy Phys. 01 (2015) 016.

[14] Y. C. Tang, K. Wildermuth, and L. D. Pearlstein, Cluster model calculation on the energy levels of the Lithium isotopes, Phys. Rev. 123, 548 (1961).

[15] S. Elhatisari, D. Lee, G. Rupak, E. Epelbaum, H. Krebs, T. A. Lähde, T. Luu, and U. G. Meißner, Ab initio alphaalpha scattering, Nature (London) 528, 111 (2015).

[16] A. Diaz-Torres and M. Wiescher, Quantifying the ${ }^{12} \mathrm{C}+{ }^{12} \mathrm{C}$ sub-Coulomb fusion with the Time-dependent wave-packet method, AIP Conf. Proc. 1491, 273 (2012).

[17] A. J. Maciejewski, Reduction, relative equilibria and potential in the two rigid bodies problem, Celest. Mech. Dyn. Astron. 63, 1 (1995).

[18] S. Krusch, Homotopy of rational maps and the quantization of skyrmions, Ann. Phys. (Amsterdam) 304, 103 (2003).

[19] D. Finkelstein and J. Rubinstein, Connection between spin, statistics, and kinks, J. Math. Phys. (N.Y.) 9, 1762 (1968).

[20] E. B. Wilson, J. C. Decius, and P. C. Cross, Molecular Vibrations (McGraw-Hill, New York, 1955).
[21] D. M. Dennison, Energy levels of the O-16 nucleus, Phys. Rev. 96, 378 (1954).

[22] C. Naya and P. Sutcliffe, Skyrmions in models with pions and rho mesons, arXiv:1803.06098.

[23] G. S. Adkins and C. R. Nappi, Stabilization of chiral solitons via vector mesons, Phys. Lett. B 137, 251 (1984).

[24] C. Barnes and N. Turok, A technique for calculating quantum corrections to solitons, arXiv:hep-th/9711071.

[25] D. R. Tilley, C. M. Cheves, J. L. Godwin, G. M. Hale, H. M. Hofmann, J. H. Kelley, C. G. Sheu, and H. R. Weller, Energy levels of light nuclei $A=5, A=6, A=7$, Nucl. Phys. A708, 3 (2002).

[26] D. T. J. Feist, P. H. C. Lau, and N. S. Manton, Skyrmions up to baryon number 108, Phys. Rev. D 87, 085034 (2013).

[27] A. A. Ogloblin, A. N. Danilov, T. L. Belyaeva, A. S. Demyanova, S. A. Goncharov, and W. Trzaska, Observation of abnormally large radii of nuclei in excited states in the vicinity of neutron thresholds, Phys. At. Nucl. 74, 1548 (2011).

[28] N. S. Manton, Lightly bound Skyrmions, tetrahedra and magic numbers, arXiv:1707.04073.

[29] C. J. Halcrow, N. S. Manton, and J. I. Rawlinson, Quantized Skyrmions from $S U(4)$ weight diagrams, Phys. Rev. C 97, 034307 (2018). 